\title{
Kirchhoff equations with Choquard exponential type nonlinearity involving the fractional Laplacian
}

\author{
Sarika Goyal* \\ Department of Mathematics, \\ Bennett University Greater Noida, Uttar Pradesh-201310, India,
} Tuhina Mukherjee ${ }^{\dagger}$

T.I.F.R. Centre for Applicable Mathematics, Post Bag No. 6503, Sharadanagar, Yelahanka New Town, Bangalore 560065.

\begin{abstract}
In this article, we deal with the existence of non-negative solutions of the class of following non local problem

$$
\left\{\begin{array}{c}
-M\left(\int_{\mathbb{R}^{n}} \int_{\mathbb{R}^{n}} \frac{|u(x)-u(y)|^{\frac{n}{s}}}{|x-y|^{2 n}} d x d y\right)(-\Delta)_{n / s}^{s} u=\left(\int_{\Omega} \frac{G(y, u)}{|x-y|^{\mu}} d y\right) g(x, u) \text { in } \Omega, \\
u=0 \text { in } \mathbb{R}^{n} \backslash \Omega,
\end{array}\right.
$$

where $(-\Delta)_{n / s}^{s}$ is the $n / s$-fractional Laplace operator, $n \geq 1, s \in(0,1)$ such that $n / s \geq 2$, $\Omega \subset \mathbb{R}^{n}$ is a bounded domain with Lipschitz boundary, $M: \mathbb{R}^{+} \rightarrow \mathbb{R}^{+}$and $g: \Omega \times \mathbb{R} \rightarrow \mathbb{R}$ are continuous functions, where $g$ behaves like $\exp \left(|u|^{\frac{n}{n-s}}\right)$ as $|u| \rightarrow \infty$.
\end{abstract}

Key words: Doubly non local problems, Kirchhoff equation, Choquard nonlinearity, Trudinger-Moser nonlinearity.

2010 Mathematics Subject Classification: 35R11, 35J60, 35A15

\section{Introduction}

Let $n \geq 1, s \in(0,1)$ such that $n / s \geq 2$ and $\Omega \subset \mathbb{R}^{n}$ be a bounded domain with Lipschitz boundary then we intend to study the existence of a non negative solutions of following fractional Kirchhoff type problem with Trudinger-Moser type Choquard nonlinearity

$$
\left\{\begin{array}{c}
-M\left(\int_{\mathbb{R}^{n}} \int_{\mathbb{R}^{n}} \frac{|u(x)-u(y)|^{\frac{n}{s}}}{|x-y|^{2 n}} d x d y\right)(-\Delta)_{n / s}^{s} u=\left(\int_{\Omega} \frac{G(y, u)}{|x-y|^{\mu}} d y\right) g(x, u) \text { in } \Omega, \\
u=0 \text { in } \mathbb{R}^{n} \backslash \Omega,
\end{array}\right.
$$

*email: sarika.goyal@bennett.edu.in

†email: tuhina@tifrbng.res.in, 
where $(-\Delta)_{n / s}^{s}$ is the $n / s$-fractional Laplace operator which, up to a normalizing constant, is defined as

$$
(-\Delta)_{n / s}^{s} u(x)=2 \lim _{\epsilon \rightarrow 0^{+}} \int_{\mathbb{R}^{n} \backslash B_{\epsilon}(x)} \frac{|u(x)-u(y)|^{\frac{n}{s}-2}(u(x)-u(y))}{|x-y|^{2 n}} d y, \quad x \in \mathbb{R}^{n}, u \in C_{0}^{\infty}\left(\mathbb{R}^{n}\right) .
$$

The functions $M: \mathbb{R}^{+} \rightarrow \mathbb{R}^{+}$and $g: \Omega \times \mathbb{R} \rightarrow \mathbb{R}$ are continuous satisfying some appropriate conditions which will be stated later on.

Our problem $(\mathcal{M})$ is basically driven by the Hardy-Littlewood-Sobolev inequality and the Trudinger-Moser inequality. Let us first recall the following well known Hardy-LittlewoodSobolev inequality [Theorem 4.3, p.106] [13].

Proposition 1.1 (Hardy-Littlewood-Sobolev inequality) Let $t, r>1$ and $0<\mu<n$ with $1 / t+\mu / n+1 / r=2, g \in L^{t}\left(\mathbb{R}^{n}\right)$ and $h \in L^{r}\left(\mathbb{R}^{n}\right)$. Then there exists a sharp constant $C(t, n, \mu, r)$, independent of $g, h$ such that

$$
\int_{\mathbb{R}^{n}} \int_{\mathbb{R}^{n}} \frac{g(x) h(y)}{|x-y|^{\mu}} \mathrm{d} x \mathrm{~d} y \leq C(t, n, \mu, r)\|g\|_{L^{t}\left(\mathbb{R}^{n}\right)}\|h\|_{L^{r}\left(\mathbb{R}^{n}\right)} .
$$

If $t=r=\frac{2 n}{2 n-\mu}$ then

$$
C(t, n, \mu, r)=C(n, \mu)=\pi^{\frac{\mu}{2}} \frac{\Gamma\left(\frac{n}{2}-\frac{\mu}{2}\right)}{\Gamma\left(n-\frac{\mu}{2}\right)}\left\{\frac{\Gamma\left(\frac{n}{2}\right)}{\Gamma(n)}\right\}^{-1+\frac{\mu}{n}} .
$$

In this case there is equality in (1.1) if and only if $g \equiv($ constant $) h$ and

$$
h(x)=A\left(\gamma^{2}+|x-a|^{2}\right)^{\frac{-(2 n-\mu)}{2}}
$$

for some $A \in \mathbb{C}, 0 \neq \gamma \in \mathbb{R}$ and $a \in \mathbb{R}^{n}$.

The study of Choquard equations originates from the work of S. Pekar [19] and P. Choquard [12] where they used elliptic equations with Hardy-Littlewood-Sobolev type nonlinearity to describe the quantum theory of a polaron at rest and to model an electron trapped in its own hole in the Hartree-Fock theory, respectively. For more details on the application of Choquard equations, we refer [17]. On the other hand, the boundary value problems involving Kirchhoff equations arise in several physical and biological systems. These type of non-local problems were initially observed by Kirchhoff in 1883 in the study of string or membrane vibrations to describe the transversal oscillations of a stretched string, particularly, taking into account the subsequent change in string length caused by oscillations.

Lü [14] in 2015 studied the following Kirchhoff problem with Choquard nonlinearity

$$
-\left(a+b \int_{\mathbb{R}^{3}}|\nabla u|^{2} d x\right) \Delta u+(1+\mu g(x)) u=\left.\left(|x|^{-\alpha} *|u|^{p}\right) u\right|^{p-2} u \text { in } \mathbb{R}^{3}
$$

for $a>0, b \geq 0, \alpha \in(0,3), p \in(2,6-\alpha), \mu>0$ is a parameter and $g$ is a nonnegative continuous potential with some growth assumptions. He proved the existence of solution to 
the above problem for $\mu$ sufficiently large and also showed their concentration behavior when $\mu$ approaches $+\infty$. In [11, authors discuss the existence and concentration of sign-changing solutions to a class of Kirchhoff-type systems with Hartree-type nonlinearity in $\mathbb{R}^{3}$ by the minimization argument on the sign-changing Nehari manifold and a quantitative deformation lemma. In the nonlocal case that is problems involving the fractional Laplace operator, Kirchhoff problem with Choquard nonlinearity has been studied by Pucci et al. in [21] via variational techniques.

The study of elliptic equations involving nonlinearity with exponential growth are motivated by the following Trudinger-Moser inequality in [15], namely

Theorem 1.2 let $\Omega$ be a open bounded domain then we define $\tilde{W}_{0}^{s, n / s}(\Omega)$ as the completion of $C_{c}^{\infty}(\Omega)$ with respect to the norm $\|u\|^{\frac{n}{s}}=\int_{\mathbb{R}^{n}} \int_{\mathbb{R}^{n}} \frac{|u(x)-u(y)|^{\frac{n}{s}}}{|x-y|^{2 n}} d x d y$. Then there exists a positive constant $\alpha_{n, s}$ given by

$$
\alpha_{n, s}=\frac{n}{\omega_{n-1}}\left(\frac{\Gamma\left(\frac{n-s}{2}\right)}{\Gamma(s / 2) 2^{s} \pi^{n / 2}}\right)^{-\frac{n}{n-s}},
$$

where $\omega_{n-1}$ be the surface area of the unit sphere in $\mathbb{R}^{n}$ and $C_{n, s}$ depending only on $n$ and $s$ such that

$$
\sup _{u \in \tilde{W}_{0}^{s, n / s}(\Omega),\|u\| \leq 1} \int_{\Omega} \exp \left(\alpha|u|^{\frac{n}{n-s}}\right) d x \leq C_{n, s}|\Omega|
$$

for each $\alpha \in\left[0, \alpha_{n, s}\right]$. Moreover there exists a $\alpha_{n, s}^{*} \geq \alpha_{n, s}$ such that the right hand side of (1.2) is $+\infty$ for $\alpha>\alpha_{n, s}^{*}$.

It is proved in [18] (see Proposition 5.2) that

$$
\alpha_{n, s}^{*}=n\left(\frac{2\left(n \mathcal{W}_{n}\right)^{2} \Gamma\left(\frac{n}{s}+1\right)}{n !} \sum_{i=0}^{\infty} \frac{(n+i-1) !}{i !(n+2 i)^{\frac{n}{s}}}\right)^{\frac{s}{n-s}},
$$

where $\mathcal{W}_{n}=\frac{w_{n-1}}{n}$ is the volume of the unit sphere in $\mathbb{R}^{n}$. It is still unknown whether $\alpha_{n, s}^{*}=\alpha_{n, s}$ or not.

The $p$-fractional Kirchhoff problems involving the Trudinger-Moser type nonlinearity has been recently addressed in [16, 23]. We also refer [6, 7] to the readers, in the linear case i.e. when $p=2$. The Choquard equations with exponential type nonlinearities has been comparatively less attended. In this regard, we cite [1] where authors studied a singularly perturbed nonlocal Schrödinger equation via variational techniques. We also refer [2] for reference. On a similar note, there is no literature available on Kirchhoff problems involving the Choquard exponential nonlinearity except the very recent article [3] where authors studied the existence of positive solutions to the following problem

$$
-m\left(\int_{\Omega}|\nabla u|^{n} d x\right) \Delta_{n} u=\left(\int_{\Omega} \frac{F(y, u)}{|x-y|^{\mu}} d y\right) f(x, u), u>0 \text { in } \Omega, u=0 \text { in } \partial \Omega
$$


where $-\Delta_{n}=\nabla \cdot\left(|\nabla u|^{n-2} \nabla u\right), \mu \in(0, n), n \geq 2, m$ and $f$ are continuous functions satisfying some additional assumptions, using the concentration compactness arguments. They also established multiplicity result corresponding to a perturbed problem via minimization over suitable subsets of Nehari manifold. Whereas in the $p$-fractional laplacian case, motivated by above research, our paper represents the first article to consider the Kirchhoff problem with Choquard exponential nonlinearity.

The problem of the type $(\mathcal{M})$ are categorized under doubly nonlocal problems because

of the presence of the term $M\left(\int_{\mathbb{R}^{n}} \int_{\mathbb{R}^{n}} \frac{|u(x)-u(y)|^{\frac{n}{s}}}{|x-y|^{2 n}} d x d y\right)$ and $\left(\int_{\Omega} \frac{G(y, u)}{|x-y|^{\mu}} d y\right) g(x, u)$ which does not allow the problem $(\mathcal{M})$ to be a pointwise identity. Additionally, we also deal with the degenerate case of Kirchhoff problem which is a new result even in the case of $s=1$. This phenomenon arises mathematical difficulties which makes the study of such a class of problem interesting. Generally, the main difficulty encountered in Kirchhoff problems is the competition between the growths of $M$ and $g$. Precisely, mere weak limit of a Palais Smale (PS) sequence is not enough to claim that it is a weak solution to $(\mathcal{M})$ because of presence of the function $M$, which holds in the case of $M \equiv 1$. Next technical hardship emerge while proving convergence of the Choquard term with respect to (PS) sequence. We use delicate ideas in Lemma 3.4 and Lemma 3.5 to establish it. Following a variational approach, we prove that the corresponding energy functional to $(\mathcal{M})$ satisfies the Mountain pass geometry and the Mountain pass critical level stays below a threshold (see Lemma 3.3) using the Moser type functions established by Parini and Ruf in [18. Then we perform a convergence analysis of the Choquard term with respect to the (PS)-sequences in Lemma 3.4. This along with the higher integrability Lemma 2.5 benefited us to get the weak limit of (PS)-sequence as a weak solution of $(\mathcal{M})$ leading to build the proof of our main result. The approach although may not be completely new but the problem is comprehensively afresh.

Our article is divided into 3 sections- Section 2 illustrates the functional set up to study $(\mathcal{M})$ and contains the main result that we intend to establish. Section 3 contains the proof of our main result.

\section{Functional Setting and Main result}

Let us consider the usual fractional Sobolev space

$$
W^{s, p}(\Omega):=\left\{u \in L^{p}(\Omega) ; \frac{(u(x)-u(y))}{|x-y|^{\frac{n}{p}+s}} \in L^{p}(\Omega \times \Omega)\right\}
$$

endowed with the norm

$$
\|u\|_{W^{s, p}(\Omega)}=\|u\|_{L^{p}(\Omega)}+\left(\int_{\Omega} \int_{\Omega} \frac{|u(x)-u(y)|^{p}}{|x-y|^{n+p s}} d x d y\right)^{\frac{1}{p}}
$$


where $\Omega \subset \mathbb{R}^{n}$ is an open set. We denote $W_{0}^{s, p}(\Omega)$ as the completion of the space $C_{c}^{\infty}(\Omega)$ with respect to the norm $\|\cdot\|_{W^{s, p}(\Omega)}$. To study fractional Sobolev spaces in details we refer to [5]. Now we define

$$
X_{0}=\left\{u \in W^{s, n / s}\left(\mathbb{R}^{n}\right): u=0 \text { in } \mathbb{R}^{n} \backslash \Omega\right\}
$$

with respect to the norm

$$
\|u\|_{X_{0}}=\left(\int_{\mathbb{R}^{n}} \int_{\mathbb{R}^{n}} \frac{|u(x)-u(y)|^{\frac{n}{s}}}{|x-y|^{2 n}} d x d y\right)^{\frac{s}{n}}=\left(\int_{Q} \frac{|u(x)-u(y)|^{\frac{n}{s}}}{|x-y|^{2 n}} d x d y\right)^{\frac{s}{n}}
$$

where $Q=\mathbb{R}^{2 n} \backslash(\mathcal{C} \Omega \times \mathcal{C} \Omega)$ and $\mathcal{C} \Omega:=\mathbb{R}^{n} \backslash \Omega$. Then $X_{0}$ is a reflexive Banach space and continuously embedded in $W_{0}^{s, p}(\Omega)$. Also $X_{0} \hookrightarrow \hookrightarrow L^{q}(\Omega)$ compactly for each $q \in[1, \infty)$. Note that the norm $\|\cdot\|_{X_{0}}$ involves the interaction between $\Omega$ and $\mathbb{R}^{n} \backslash \Omega$. We denote $\|\cdot\|_{X_{0}}$ by $\|$.$\| in$ future, for notational convenience. This type of functional setting was introduced by Servadei and Valdinoci for $p=2$ in [22] and for $p \neq 2$ in 8 ].

Moreover, we define the space

$$
\tilde{W}_{0}^{s, p}(\Omega)=\overline{C_{0}(\Omega)}\|\cdot\|_{W^{s, p}\left(\mathbb{R}^{n}\right)}
$$

The space $\tilde{W}_{0}^{s, p}(\Omega)$ is equivalent to the completion of $C_{0}^{\infty}(\Omega)$ with respect to the semi norm $\int_{\mathbb{R}^{n}} \int_{\mathbb{R}^{n}} \frac{|u(x)-u(y)|^{\frac{n}{s}}}{|x-y|^{2 n}} d x d y$ (see for example [ [9], Remark 2.5]). If $\partial \Omega$ is Lipschitz, then $\tilde{W}_{0}^{s, p}(\Omega)=X_{0}$, (see[[10], Proposition B.1]). The embedding $W_{0}^{s, \frac{n}{s}}(\Omega) \ni u \longmapsto \exp \left(|u|^{\beta}\right) \in$ $L^{1}(\Omega)$ is compact for all $\beta \in\left(1, \frac{n}{n-s}\right)$ and is continuous when $\beta=\frac{n}{n-s}$.

We now state our assumptions on $M$ and $g$. The function $M: \mathbb{R}^{+} \rightarrow \mathbb{R}^{+}$is a continuous function which satisfies the following assumptions:

$(M 1)$ For all $t, s \geq 0$, it holds

$$
\hat{M}(t+s) \geq \hat{M}(t)+\hat{M}(s)
$$

where $\hat{M}(t)=\int_{0}^{t} M(s) d s$, the primitive of $M$.

(M2) There exists a $\gamma>1$ such that $t \mapsto \frac{M(t)}{t^{\gamma-1}}$ is non increasing for each $t>0$.

(M3) For each $b>0$, there exists a $\kappa:=\kappa(b)>0$ such that $M(t) \geq \kappa$ whenever $t \geq b$.

The condition (M3) asserts that the function $M$ has possibly a zero only when $t=0$.

Remark 2.1 From (M2), we can easily deduce that $\gamma \hat{M}(t)-M(t) t$ is non decreasing for $t>0$ and

$$
\gamma \hat{M}(t)-M(t) t \geq 0 \quad \forall t \geq 0
$$

We also have the following remark as a consequence of (2.1). 
Remark 2.2 For each $t \geq 0$, by using (2.1) we have

$$
\frac{d}{d t}\left(\frac{\hat{M}(t)}{t^{\gamma}}\right)=\frac{M(t)}{t^{\gamma}}-\frac{\gamma \hat{M}(t)}{t^{\gamma+1}} \leq 0
$$

So the map $t \mapsto \frac{\hat{M}(t)}{t^{\gamma}}$ is non increasing for $t>0$. Hence

$$
\hat{M}(t) \geq \hat{M}(1) t^{\gamma} \text { for all } t \in[0,1]
$$

and

$$
\hat{M}(t) \leq \hat{M}(1) t^{\gamma} \text { for all } t \geq 1
$$

We note that the condition $(M 1)$ is valid whenever $M$ is non decreasing.

Example 1 Let $M(t)=m_{0}+a t^{\gamma-1}$, where $m_{0}, a \geq 0$ and $\gamma>1$ such that $m_{0}+a>0$ then $M$ satisfies the conditions $(M 1)-(M 3)$. If $m_{0}=0$, this forms an example of the degenerate case whereas of the non degenerate case if $m_{0}>0$.

The nonlinearity $g: \Omega \times \mathbb{R} \rightarrow \mathbb{R}$ is a continuous function such that $g(x, t)=h(x, t) \exp \left(|t|^{\frac{n}{n-s}}\right)$, where $h(x, t)$ satisfies the following assumptions:

$(g 1) h \in C^{1}(\bar{\Omega} \times \mathbb{R}), h(x, t)=0$, for all $t \leq 0, h(x, t)>0$, for all $t>0$.

$(g 2)$ For any $\epsilon>0, \lim _{t \rightarrow \infty} \sup _{x \in \bar{\Omega}} h(x, t) \exp \left(-\epsilon|t|^{\frac{n}{n-s}}\right)=0, \lim _{t \rightarrow \infty} \inf _{x \in \bar{\Omega}} h(x, t) \exp \left(\epsilon|t|^{\frac{n}{n-s}}\right)=\infty$.

(g3) There exist positive constants $T, T_{0}$ and $\gamma_{0}$ such that

$$
0<t^{\gamma_{0}} G(x, t) \leq T_{0} g(x, t) \text { for all }(x, t) \in \Omega \times\left[t_{0},+\infty\right) .
$$

(g4) For $\gamma>1$ (defined in (M2)), there exists a $l>\frac{\gamma n}{2 s}-1$ such that the map $t \mapsto \frac{g(x, t)}{t^{l}}$ is increasing on $\mathbb{R}^{+} \backslash\{0\}$, uniformly in $x \in \Omega$.

Remark 2.3 Condition (g4) implies that for each $x \in \Omega$,

$$
t \mapsto \frac{g(x, t)}{t^{\frac{\gamma n}{2 s}-1}} \text { is increasing for } t>0 \text { and } \lim _{t \rightarrow 0^{+}} \frac{g(x, t)}{t^{\frac{\gamma n}{2 s}-1}}=0
$$

uniformly in $x \in \Omega$. Also, for each $(x, t) \in \Omega \times \mathbb{R}$ we have

$$
(l+1) G(x, t) \leq \operatorname{tg}(x, t) .
$$

Example 2 Let $g(x, t)=h(x, t) e^{|t|^{\frac{n}{n-s}}}$, where $h(x, t)=\left\{\begin{array}{l}0 \text { if } t \leq 0 \\ t^{\alpha+\left(\frac{\gamma n}{2 s}-1\right)} \exp \left(d t^{\beta}\right) \text { if } t>0 .\end{array}\right.$ for some $\alpha>0,0<d \leq \alpha_{n, s}$ and $1 \leq \beta<\frac{n}{n-s}$. Then $g$ satisfies all the conditions from $(g 1)-(g 4)$. 
Definition 2.4 We say that $u \in X_{0}$ is a weak solution of $(\mathcal{M})$ if, for all $\phi \in X_{0}$, it satisfies $M\left(\|u\|^{\frac{n}{s}}\right) \int_{\mathbb{R}^{2 n}} \frac{|u(x)-u(y)|^{\frac{n}{s}-2}(u(x)-u(y))(\phi(x)-\phi(y))}{|x-y|^{2 n}} d x d y=\int_{\Omega}\left(\int_{\Omega} \frac{G(y, u)}{|x-y|^{\mu}} d y\right) g(x, u) \phi d x$.

Before stating our main Theorem, we recall a result of 18 which will be used to find an upper bound for the Mountain Pass critical level. Assume that $0 \in \Omega$ and $B_{1}(0) \subset \Omega$. Then we consider the following Moser type functions which is given by equation (5.2) of [18]. For each $x \in \mathbb{R}^{n}$ and $k \in \mathbb{N}$,

$$
\tilde{w}_{k}(x)=\left\{\begin{array}{l}
|\log k|^{\frac{n-s}{n}}, \quad \text { if } 0 \leq|x| \leq \frac{1}{k}, \\
\frac{|\log (|x|)|}{|\log (1 / k)|^{s / n}}, \quad \text { if } \frac{1}{k} \leq|x| \leq 1, \\
0, \text { if }|x| \geq 1,
\end{array}\right.
$$

then $\operatorname{supp}\left(\tilde{w}_{k}\right) \subset B_{1}(0) \subset \Omega$ and $\left.\tilde{w}_{k}\right|_{B_{1}(0)} \in W_{0}^{s, \frac{n}{s}}\left(B_{1}(0)\right)$.

Now by Proposition 5.1 of [18] we know that

$$
\lim _{k \rightarrow \infty}\left\|\tilde{w}_{k}\right\|^{\frac{n}{s}}=\lim _{k \rightarrow \infty} \int_{\mathbb{R}^{n}} \int_{\mathbb{R}^{n}} \frac{\left|\tilde{w}_{k}(x)-\tilde{w}_{k}(y)\right|^{\frac{n}{s}}}{|x-y|^{2 n}} d x d y=\gamma_{n, s}
$$

where

$$
\gamma_{n, s}:=\frac{2\left(n \mathcal{W}_{n}\right)^{2} \Gamma\left(\frac{n}{s}+1\right)}{n !} \sum_{i=0}^{\infty} \frac{(n+i-1) !}{i !(n+2 i)^{\frac{n}{s}}} .
$$

where $\mathcal{W}_{n}$ denotes the volume of $n$-dimensional unit sphere. We also recall the following result of Lions known as higher integrability Lemma in case of fractional Laplacian, proved in [20].

Lemma 2.5 Let $\left\{v_{k}:\left\|v_{k}\right\|=1\right\}$ be a sequence in $W_{0}^{s, n / s}(\Omega)$ converging weakly to a non-zero function $v$. Then for every $p$ such that $p<\alpha_{n, s}\left(1-\|v\|^{\frac{n}{s}}\right)^{\frac{-s}{n-s}}$,

$$
\sup _{k} \int_{\Omega} \exp \left(p\left|v_{k}\right|^{\frac{n}{n-s}}\right)<+\infty \text {. }
$$

Now we state our main result:

Theorem 2.6 Suppose $(M 1)-(M 3)$ and $(g 1)-(g 4)$ hold. Assume in addition that for $\beta>\frac{2 \alpha_{n, s}^{*}}{\alpha_{n, s}}$,

$$
\lim _{t \rightarrow+\infty} \frac{\operatorname{tg}(x, t) G(x, t)}{\exp \left(\beta t^{\frac{n}{n-s}}\right)}=\infty \text { uniformly in } x \in \bar{\Omega} .
$$

Then, problem $(\mathcal{M})$ admit a non negative non trivial solution. 


\section{Proof of Main result}

We begin this section with the study of mountain pass structure and Palais-Smale sequences corresponding to the energy functional $J: X_{0} \rightarrow \mathbb{R}$ associated to the problem $(\mathcal{M})$ which is defined as

$$
J(u)=\frac{s}{n} \hat{M}\left(\|u\|^{\frac{n}{s}}\right)-\frac{1}{2} \int_{\Omega}\left(\int_{\Omega} \frac{G(y, u)}{|x-y|^{\mu}} d y\right) G(x, u) d x .
$$

From the assumptions, $(g 1)-(g 4)$, we obtain that for any $\epsilon>0, r \geq 1,1 \leq \alpha<l+1$ there exists $C(\epsilon)>0$ such that

$$
|G(x, t)| \leq \epsilon|t|^{\alpha}+C(\epsilon)|t|^{r} \exp \left((1+\epsilon)|t|^{\frac{n}{n-s}}\right), \text { for all }(x, t) \in \Omega \times \mathbb{R} .
$$

Now by Proposition 1.1, for any $u \in X_{0}$ we obtain

$$
\int_{\Omega}\left(\int_{\Omega} \frac{G(y, u)}{|x-y|^{\mu}} d y\right) G(x, u) d x \leq C(n, \mu)\|G(\cdot, u)\|_{L^{2 n-\mu}(\Omega)}^{2} .
$$

This implies that $J$ is well defined using Theorem 1.2. Also one can easily see that $J$ is Fréchet differentiable and the critical points of $J$ are the weak solutions of $(\mathcal{M})$.

Lemma 3.1 Assume that the conditions $(M 1)$ and $(g 1)-(g 4)$ hold. Then $J$ satisfies the Mountain Pass geometry around 0.

Proof. From (3.1), (3.2), Hölder inequality and Sobolev embedding, we have

$$
\begin{aligned}
& \int_{\Omega}\left(\int_{\Omega} \frac{G(y, u)}{|x-y|^{\mu}} d y\right) G(x, u) d x \\
& \leq C(n, \mu) 2^{2}\left(\epsilon^{\frac{2 n}{2 n-\mu}} \int_{\Omega}|u|^{\frac{2 n \alpha}{2 n-\mu}}+(C(\epsilon))^{\frac{2 n}{2 n-\mu}} \int_{\Omega}|u|^{\frac{2 r n}{2 n-\mu}} \exp \left(\frac{2 n(1+\epsilon)}{2 n-\mu}|u|^{\frac{n}{n-s}}\right)\right)^{\frac{2 n-\mu}{n}} \\
& \leq C\left(\epsilon^{\frac{2 n}{2 n-\mu}} \int_{\Omega}|u|^{\frac{2 n \alpha}{2 n-\mu}}+C_{1}(\epsilon)\|u\|^{\frac{2 r n}{2 n-\mu}}\left(\int_{\Omega} \exp \left(\frac{4 n(1+\epsilon)\|u\| \frac{n}{n-s}}{2 n-\mu}\left(\frac{|u|}{\|u\|}\right)^{\frac{n}{n-s}}\right)\right)^{\frac{1}{2}}\right)^{\frac{2 n-\mu}{n}}
\end{aligned}
$$

So if we choose $\epsilon>0$ small enough and $u$ such that $\frac{4 n(1+\epsilon)\|u\|^{\frac{n}{n-s}}}{2 n-\mu} \leq \alpha_{n, s}$ then using the fractional Trudinger-Moser inequality (1.2) in (3.3), we obtain

$$
\begin{aligned}
\int_{\Omega}\left(\int_{\Omega} \frac{G(y, u)}{|x-y|^{\mu}} d y\right) G(x, u) d x & \leq C_{2}(\epsilon)\left(\|u\|^{\frac{2 n \alpha}{2 n-\mu}}+\|u\|^{\frac{2 r n}{2 n-\mu}}\right)^{\frac{2 n-\mu}{n}} \\
& \leq C_{3}(\epsilon)\left(\|u\|^{2 \alpha}+\|u\|^{2 r}\right) .
\end{aligned}
$$

Using (2.2) and above estimate, we have

$$
J(u) \geq \frac{s}{n} \hat{M}(1)\|u\|^{\frac{\gamma n}{s}}-C_{3}(\epsilon)\left(\|u\|^{2 \alpha}+\|u\|^{2 r}\right),
$$

when $\|u\| \leq 1$. Choosing $\alpha>\frac{\gamma n}{2 s}, r>\frac{\gamma n}{2 s}$ and $\rho>0$ such that $\rho<\min \left\{1,\left(\frac{\alpha_{n, s}(2 n-\mu)}{4 n(1+\epsilon)}\right)^{\frac{n-s}{n}}\right\}$ we obtain $J(u) \geq \sigma>0$ for all $u \in X_{0}$ with $\|u\|=\rho$ and for some $\sigma>0$ depending on $\rho$. 
The condition $(g 4)$ implies that there exist some positive constants $C_{1}$ and $C_{2}$ such that

$$
G(x, t) \geq C_{1} t^{l+1}-C_{2} \text { for all }(x, t) \in \Omega \times[0, \infty) .
$$

Let $\phi \in X_{0}$ such that $\phi \geq 0$ and $\|\phi\|=1$ then by (3.4) we obtain

$$
\begin{aligned}
\int_{\Omega}\left(\int_{\Omega} \frac{G(y, t \phi)}{|x-y|^{\mu}} d y\right) G(x, t \phi) d x \geq & \int_{\Omega} \int_{\Omega} \frac{\left(C_{1}(t \phi)^{l+1}(y)-C_{2}\right)\left(C_{1}(t \phi)^{l+1}(x)-C_{2}\right)}{|x-y|^{\mu}} d x d y \\
= & C_{1}^{2} t^{2(l+1)} \int_{\Omega} \int_{\Omega} \frac{\phi^{l+1}(y) \phi^{l+1}(x)}{|x-y|^{\mu}} d x d y \\
& -2 C_{1} C_{2} t^{l+1} \int_{\Omega} \int_{\Omega} \frac{\phi^{l+1}(y)}{|x-y|^{\mu}} d x d y+C_{2}^{2} \int_{\Omega} \int_{\Omega} \frac{1}{|x-y|^{\mu}} d x d y .
\end{aligned}
$$

This together with (2.3), we obtain

$$
\begin{aligned}
J(t \phi) & \leq \frac{s}{n} M(1)\|t \phi\|^{\frac{\gamma n}{s}}-\frac{1}{2} \int_{\Omega}\left(\int_{\Omega} \frac{G(y, t \phi)}{|x-y|^{\mu}} d y\right) G(x, t \phi) d x \\
& \leq C_{3}+C_{4} t^{\frac{\gamma n}{s}}-C_{5} t^{2(l+1)}+C_{6} t^{l+1}
\end{aligned}
$$

where $C_{i}^{\prime} s$ are positive constants for $i=3,4,5,6$. This implies that $J(t \phi) \rightarrow-\infty$ as $t \rightarrow \infty$, since $l+1>\frac{\gamma n}{2 s}$. Thus there exists a $v_{0} \in X_{0}$ with $\left\|v_{0}\right\|>\rho$ such that $J\left(v_{0}\right)<0$. Therefore, $J$ satisfies Mountain Pass geometry near 0 .

Let $\Gamma=\left\{\gamma \in C\left([0,1], X_{0}\right): \gamma(0)=0, J(\gamma(1))<0\right\}$ and define the Mountain Pass critical level $\left.c_{*}=\inf _{\gamma \in \Gamma \in[0,1]} \max _{t \in[} J(t)\right)$. Then by Lemma 3.1] and the Mountain pass theorem we know that there exists a Palais Smale sequence $\left\{u_{k}\right\} \subset X_{0}$ for $J$ at $c_{*}$ that is

$$
J\left(u_{k}\right) \rightarrow c_{*} \text { and } J^{\prime}\left(u_{k}\right) \rightarrow 0 \text { as } k \rightarrow \infty
$$

Lemma 3.2 Every Palais-Smale sequence of $J$ is bounded in $X_{0}$.

Proof. Let $\left\{u_{k}\right\} \subset X_{0}$ denotes a $(P S)_{c}$ sequence of $J$ that is

$$
J\left(u_{k}\right) \rightarrow c \text { and } J^{\prime}\left(u_{k}\right) \rightarrow 0 \text { as } k \rightarrow \infty
$$

for some $c \in \mathbb{R}$. This implies

$$
\begin{aligned}
& \frac{s \hat{M}\left(\left\|u_{k}\right\|^{\frac{n}{s}}\right)}{n}-\frac{1}{2} \int_{\Omega}\left(\int_{\Omega} \frac{G\left(y, u_{k}\right)}{|x-y|^{\mu}} d y\right) G\left(x, u_{k}\right) d x \rightarrow c \text { as } k \rightarrow \infty, \\
& \mid M\left(\left\|u_{k}\right\|^{\frac{n}{s}}\right) \int_{\mathbb{R}^{n}} \int_{\mathbb{R}^{n}} \frac{\left|u_{k}(x)-u_{k}(y)\right|^{\frac{n}{s}-2}\left(u_{k}(x)-u_{k}(y)\right)(\phi(x)-\phi(y))}{|x-y|^{2 n}} d x d y \\
& -\int_{\Omega}\left(\int_{\Omega} \frac{G\left(y, u_{k}\right)}{|x-y|^{\mu}} d y\right) g\left(x, u_{k}\right) \phi d x \mid \leq \epsilon_{k}\|\phi\|
\end{aligned}
$$


where $\epsilon_{k} \rightarrow 0$ as $k \rightarrow \infty$. In particular, taking $\phi=u_{k}$ we get

$$
\left|M\left(\left\|u_{k}\right\|^{\frac{n}{s}}\right)\left\|u_{k}\right\|^{\frac{n}{s}}-\int_{\Omega}\left(\int_{\Omega} \frac{G\left(y, u_{k}\right)}{|x-y|^{\mu}} d y\right) g\left(x, u_{k}\right) u_{k} d x\right| \leq \epsilon_{k}\left\|u_{k}\right\| .
$$

Now Remark (2.3) gives us that

$$
(l+1) \int_{\Omega}\left(\int_{\Omega} \frac{G\left(y, u_{k}\right)}{|x-y|^{\mu}} d y\right) G\left(x, u_{k}\right) d x \leq \int_{\Omega}\left(\int_{\Omega} \frac{G\left(y, u_{k}\right)}{|x-y|^{\mu}} d y\right) g\left(x, u_{k}\right) u_{k} d x .
$$

Then using (3.5), (3.6) along with (3.7) and (2.1), we get

$$
\begin{aligned}
& J\left(u_{k}\right)-\frac{1}{2(l+1)}\left\langle J^{\prime}\left(u_{k}\right), u_{k}\right\rangle=\frac{s}{n} \hat{M}\left(\left\|u_{k}\right\|^{\frac{n}{s}}\right)-\frac{1}{2(l+1)} M\left(\left\|u_{k}\right\|^{\frac{n}{s}}\right)\left\|u_{k}\right\|^{\frac{n}{s}} \\
& \quad-\frac{1}{2}\left[\int_{\Omega}\left(\int_{\Omega} \frac{G\left(y, u_{k}\right)}{|x-y|^{\mu}} d y\right) G\left(x, u_{k}\right) d x-\frac{1}{(l+1)} \int_{\Omega}\left(\int_{\Omega} \frac{G\left(y, u_{k}\right)}{|x-y|^{\mu}} d y\right) g\left(x, u_{k}\right) u_{k} d x\right] \\
& \geq \frac{s \hat{M}\left(\left\|u_{k}\right\|^{\frac{n}{s}}\right)}{n}-\frac{M\left(\left\|u_{k}\right\|^{\frac{n}{s}}\right)\left\|u_{k}\right\|^{\frac{n}{s}}}{2(l+1)} \\
& \geq\left(\frac{s}{n \gamma}-\frac{1}{2(l+1)}\right) M\left(\left\|u_{k}\right\|^{\frac{n}{s}}\right)\left\|u_{k}\right\|^{\frac{n}{s}} .
\end{aligned}
$$

To prove the Lemma, we assume by contradiction that $\left\{\left\|u_{k}\right\|\right\}$ is an unbounded sequence. Then without loss of generality, we can assume that, up to a subsequence, $\left\|u_{k}\right\| \rightarrow \infty$ and $\left\|u_{k}\right\| \geq \alpha>0$ for some $\alpha$ and for all $k$. This along with (3.8) and (M3) gives us

$$
J\left(u_{k}\right)-\frac{1}{2(l+1)}\left\langle J^{\prime}\left(u_{k}\right), u_{k}\right\rangle \geq\left(\frac{s}{n \gamma}-\frac{1}{2(l+1)}\right) \kappa\left\|u_{k}\right\|^{\frac{n}{s}}
$$

where $\kappa$ depends on $\alpha$. Also from (3.5) and (3.6) it follows that

$$
J\left(u_{k}\right)-\frac{1}{2(l+1)}\left\langle J^{\prime}\left(u_{k}\right), u_{k}\right\rangle \leq C\left(1+\epsilon_{k} \frac{\left\|u_{k}\right\|}{2(l+1)}\right)
$$

for some constant $C>0$. Therefore from (3.9) and $(3.10)$ we get that

$$
\left(\frac{s}{n \gamma}-\frac{1}{2(l+1)}\right) \kappa\left\|u_{k}\right\|^{\frac{n}{s}} \leq C\left(1+\epsilon_{k} \frac{\left\|u_{k}\right\|}{2(l+1)}\right)
$$

which gives a contradiction because $l+1>\frac{\gamma n}{2 s}$ and $\frac{n}{s}>1$. This implies that $\left\{u_{k}\right\}$ must be bounded in $X_{0}$.

Assume that $0 \in \Omega$ and $\rho>0$ be such that $B_{\rho}(0) \subset \Omega$. Then for $x \in \mathbb{R}^{n}$, we define $w_{k}(x):=\tilde{w}_{k}\left(\frac{x}{\rho}\right)$, where $\tilde{w}_{k}$ is same as (2.4) then $\operatorname{supp}\left(w_{k}\right) \in B_{\rho}(0) \subset \Omega$. We note that $w_{k} \in W_{0}^{s, \frac{n}{s}}\left(\mathbb{R}^{n}\right)$ and by (2.5), we have

$$
\lim _{k \rightarrow \infty}\left\|w_{k}\right\|^{\frac{n}{s}}=\lim _{k \rightarrow \infty} \int_{\mathbb{R}^{n}} \int_{\mathbb{R}^{n}} \frac{\left|\tilde{w}_{k}(x)-\tilde{w}_{k}(y)\right|^{\frac{n}{s}}}{|x-y|^{2 n}} d x d y=\gamma_{n, s} .
$$

Next, we use $w_{k}$ 's efficiently to obtain the following bound on $c_{*}$. 
Lemma 3.3 It holds that

$$
0<c_{*}<\frac{s}{n} \hat{M}\left(\left(\frac{2 n-\mu}{2 n} \alpha_{n, s}\right)^{\frac{n-s}{s}}\right) .
$$

Proof. Using Lemma 3.1, we deduce that $c_{*}>0$ and $J(t \phi) \rightarrow-\infty$ as $t \rightarrow \infty$ if $0 \leq \phi \in$ $X_{0} \backslash\{0\}$ with $\|\phi\|=1$. Also by definition of $c_{*}$, we have $c_{*} \leq \max _{t \in[0,1]} J(t \phi)$ for each non negative $\phi \in X_{0} \backslash\{0\}$ with $J(\phi)<0$ which assures that it is enough to prove that there exists a non negative $w \in X_{0} \backslash\{0\}$ such that

$$
\max _{t \in[0, \infty)} J(t w)<\frac{s}{n} \hat{M}\left(\left(\frac{2 n-\mu}{2 n} \alpha_{n, s}\right)^{\frac{n-s}{s}}\right) .
$$

To prove this, we consider the sequence of non negative functions $\left\{w_{k}\right\}$ (defined before this Lemma) and claim that there exists a $k \in \mathbb{N}$ such that

$$
\max _{t \in[0, \infty)} J\left(t w_{k}\right)<\frac{s}{n} \hat{M}\left(\left(\frac{2 n-\mu}{2 n} \alpha_{n, s}\right)^{\frac{n-s}{s}}\right) .
$$

Suppose this is not true, then for all $k \in \mathbb{N}$ there exists a $t_{k}>0$ such that

$$
\begin{aligned}
& \max _{t \in[0, \infty)} J\left(t w_{k}\right)=J\left(t_{k} w_{k}\right) \geq \frac{s}{n} \hat{M}\left(\left(\frac{2 n-\mu}{2 n} \alpha_{n, s}\right)^{\frac{n-s}{s}}\right) \\
& \text { and }\left.\frac{d}{d t}\left(J\left(t w_{k}\right)\right)\right|_{t=t_{k}}=0 .
\end{aligned}
$$

From the proof of Lemma 3.1, $J\left(t w_{k}\right) \rightarrow-\infty$ as $t \rightarrow \infty$ for each $k$. Then we infer that $\left\{t_{k}\right\}$ must be a bounded sequence in $\mathbb{R}$ which implies that there exists a $t_{0}$ such that, up to a subsequence which we still denote by $\left\{t_{k}\right\}, t_{k} \rightarrow t_{0}$ as $k \rightarrow \infty$. From (3.12) and definition of $J\left(t_{k} w_{k}\right)$ we obtain

$$
\frac{s}{n} \hat{M}\left(\left(\frac{2 n-\mu}{2 n} \alpha_{n, s}\right)^{\frac{n-s}{s}}\right)<\frac{s}{n} \hat{M}\left(\left\|t_{k} w_{k}\right\|^{\frac{n}{s}}\right) .
$$

Since $\hat{M}$ is monotone increasing, from (3.13) we get that

$$
\left\|t_{k} w_{k}\right\|^{\frac{n}{s}} \geq\left(\frac{2 n-\mu}{2 n} \alpha_{n, s}\right)^{\frac{n-s}{s}} .
$$

From (3.14) and since (3.11) holds, we infer that

$$
t_{k}(\log k)^{\frac{n-s}{n}} \rightarrow \infty \text { as } k \rightarrow \infty .
$$

Furthermore from (3.12), we have

$$
\begin{aligned}
M\left(\left\|t_{k} w_{k}\right\|^{\frac{n}{s}}\right)\left\|t_{k} w_{k}\right\|^{\frac{n}{s}} & =\int_{\Omega}\left(\int_{\Omega} \frac{G\left(y, t_{k} w_{k}\right)}{|x-y|^{\mu}} d y\right) g\left(x, t_{k} w_{k}\right) t_{k} w_{k} d x \\
& \geq \int_{B_{\rho / k}} g\left(x, t_{k} w_{k}\right) t_{k} w_{k} \int_{B_{\rho / k}} \frac{G\left(y, t_{k} w_{k}\right)}{|x-y|^{\mu}} d y d x .
\end{aligned}
$$


In addition, as in equation (2.11) p. 1943 in [1], it is easy to get that

$$
\int_{B_{\rho / k}} \int_{B_{\rho / k}} \frac{d x d y}{|x-y|^{\mu}} \geq C_{\mu, n}\left(\frac{\rho}{k}\right)^{2 n-\mu},
$$

where $C_{\mu, n}$ is a positive constant depending on $\mu$ and $n$. From (2.6), it is easy to deduce that for $\beta>\frac{2 \alpha_{n, s}^{*}}{\alpha_{n, s}}$ and for each $d>0$ there exists a $r_{d} \in \mathbb{N}$ such that

$$
r g(x, r) G(x, r) \geq d \exp \left(\beta|r|^{\frac{n}{n-s}}\right) \text { whenever } r \geq r_{d} .
$$

Since (3.15) holds, we can choose a $N_{d} \in \mathbb{N}$ such that

$$
t_{k}(\log k)^{\frac{n-s}{n}} \geq r_{d} \text { for all } k \geq N_{d} .
$$

Using these estimates in (3.16) and from (3.14), for $d$ large enough we get that

$$
\begin{aligned}
M\left(\left\|t_{k} w_{k}\right\|^{\frac{n}{s}}\right)\left\|t_{k} w_{k}\right\|^{\frac{n}{s}} & \geq d \exp \left(\beta t_{k}^{\frac{n}{n-s}}|\log k|\right) C_{\mu, n}\left(\frac{\rho}{k}\right)^{2 n-\mu} \\
& =d C_{\mu, n} \rho^{2 n-\mu} \exp \left(\left(\beta t_{k}^{\frac{n}{n-s}}-(2 n-\mu)\right) \log k\right) \\
& \geq d C_{\mu, n} \rho^{2 n-\mu} \exp \left(\log k\left(\frac{(2 n-\mu) \beta \alpha_{n, s}}{2 n\left\|w_{k}\right\|^{\frac{n}{n-s}}}-(2 n-\mu)\right)\right)
\end{aligned}
$$

Since $\beta>\frac{2 \alpha_{n, s}^{*}}{\alpha_{n, s}}=\frac{2 n \gamma_{n, s}^{\frac{s}{n-s}}}{\alpha_{n, s}}$ and (3.11) hold, the R.H.S. of (3.17) tends to $+\infty$ as $k \rightarrow \infty$. Whereas from continuity of $M$ it follows that

$$
\lim _{k \rightarrow \infty} M\left(\left\|t_{k} w_{k}\right\|^{\frac{n}{s}}\right)\left\|t_{k} w_{k}\right\|^{\frac{n}{s}}=M\left(t_{0}^{\frac{n}{s}} \gamma_{n, s}\right)\left(t_{0}^{\frac{n}{s}} \gamma_{n, s}\right),
$$

which is a contradiction. This establishes our claim and we conclude the proof of Lemma.

In order to prove that a Palais-Smale sequence converges to a weak solution of problem $(\mathcal{M})$, we need the following convergence Lemma. The idea of proof is borrowed from Lemma 2.4 in [1].

Lemma 3.4 If $\left\{u_{k}\right\}$ is a Palais Smale sequence for $J$ at $c$ then there exists a $u \in X_{0}$ such that, up to a subsequence.

$$
\left(\int_{\Omega} \frac{G\left(y, u_{k}\right)}{|x-y|^{\mu}} d y\right) G\left(x, u_{k}\right) \rightarrow\left(\int_{\Omega} \frac{G(y, u)}{|x-y|^{\mu}} d y\right) G(x, u) \text { in } L^{1}(\Omega)
$$

Proof. From Lemma 3.2, we know that the sequence $\left\{u_{k}\right\}$ must be bounded in $X_{0}$. Consequently, up to a subsequence, there exists a $u \in X_{0}$ such that $u_{k} \rightarrow u$ weakly in $X_{0}$ and strongly in $L^{q}(\Omega)$ for any $q \in[1, \infty)$ as $k \rightarrow \infty$. Also, still up to a subsequence, we can assume that $u_{k}(x) \rightarrow u(x)$ pointwise a.e. for $x \in \Omega$.

From (3.5), (3.6) and (3.7) we get that there exists a constant $C>0$ such that

$$
\begin{gathered}
\int_{\Omega}\left(\int_{\Omega} \frac{G\left(y, u_{k}\right)}{|x-y|^{\mu}} d y\right) G\left(x, u_{k}\right) d x \leq C, \\
\int_{\Omega}\left(\int_{\Omega} \frac{G\left(y, u_{k}\right)}{|x-y|^{\mu}} d y\right) g\left(x, u_{k}\right) u_{k} d x \leq C .
\end{gathered}
$$


Now, it is well known that if $f \in L^{1}(\Omega)$ then for any $\epsilon>0$ there exists a $\delta>0$ such that

$$
\left|\int_{U} f(x) d x\right|<\epsilon
$$

for any measurable set $U \subset \Omega$ with $|U| \leq \delta$. Also $f \in L^{1}(\Omega)$ implies that for any fixed $\delta>0$ there exists $M>0$ such that

$$
|\{x \in \Omega:|f(x)| \geq M\}| \leq \delta .
$$

Now using (3.19), we have

$$
\left(\int_{\Omega} \frac{G\left(y, u_{k}\right)}{|x-y|^{\mu}} d y\right) G\left(\cdot, u_{k}\right) \in L^{1}(\Omega)
$$

and also by (3.2)

$$
\left(\int_{\Omega} \frac{G(y, u)}{|x-y|^{\mu}} d y\right) G(\cdot, u) \in L^{1}(\Omega)
$$

Now we fix $\delta>0$ and choose $M>\max \left\{\left(\frac{C T_{0}}{\delta}\right)^{\frac{1}{\gamma_{0}+1}}, t_{0}\right\}$. Then we use $(g 3)$ to obtain

$$
\begin{aligned}
\int_{\Omega \cap\left\{u_{k} \geq M\right\}} & \left(\int_{\Omega} \frac{G\left(y, u_{k}\right)}{|x-y|^{\mu}} d y\right) G\left(x, u_{k}\right) d x \leq T_{0} \int_{\Omega \cap\left\{u_{k} \geq M\right\}}\left(\int_{\Omega} \frac{G\left(y, u_{k}\right)}{|x-y|^{\mu}} d y\right) \frac{g\left(x, u_{k}\right)}{u_{k}^{\gamma_{0}}} d x \\
& \leq \frac{T_{0}}{M^{\gamma_{0}+1}} \int_{\Omega \cap\left\{u_{k} \geq M\right\}}\left(\int_{\Omega} \frac{G\left(y, u_{k}\right)}{|x-y|^{\mu}} d y\right) g\left(x, u_{k}\right) u_{k} d x<\delta .
\end{aligned}
$$

Next we consider

$$
\begin{aligned}
& \left|\int_{\Omega}\left(\int_{\Omega} \frac{G\left(y, u_{k}\right)}{|x-y|^{\mu}} d y\right) G\left(x, u_{k}\right) d x-\int_{\Omega}\left(\int_{\Omega} \frac{G(y, u)}{|x-y|^{\mu}} d y\right) G(x, u) d x\right| \\
& \leq 2 \delta+\left|\int_{\Omega \cap\left\{u_{k} \leq M\right\}}\left(\int_{\Omega} \frac{G\left(y, u_{k}\right)}{|x-y|^{\mu}} d y\right) G\left(x, u_{k}\right) d x-\int_{\Omega \cap\{u \leq M\}}\left(\int_{\Omega} \frac{G(y, u)}{|x-y|^{\mu}} d y\right) G(x, u) d x\right|
\end{aligned}
$$

To prove the result, it is enough to establish that as $k \rightarrow \infty$

$$
\int_{\Omega \cap\left\{u_{k} \leq M\right\}}\left(\int_{\Omega} \frac{G\left(y, u_{k}\right)}{|x-y|^{\mu}} d y\right) G\left(x, u_{k}\right) d x \rightarrow \int_{\Omega \cap\{u \leq M\}}\left(\int_{\Omega} \frac{G(y, u)}{|x-y|^{\mu}} d y\right) G(x, u) d x .
$$

Since $\left(\int_{\Omega} \frac{G(y, u)}{|x-y|^{\mu}} d y\right) G(\cdot, u) \in L^{1}(\Omega)$, so by Fubini's theorem we get

$$
\begin{aligned}
& \lim _{K \rightarrow \infty} \int_{\Omega \cap\{u \leq M\}}\left(\int_{\Omega \cap\{u \geq K\}} \frac{G(y, u)}{|x-y|^{\mu}} d y\right) G(x, u) d x \\
& =\lim _{K \rightarrow \infty} \int_{\Omega \cap\{u \geq K\}}\left(\int_{\Omega \cap\{u \leq M\}} \frac{G(y, u)}{|x-y|^{\mu}} d y\right) G(x, u) d x=0 .
\end{aligned}
$$


Thus we can fix a $K>\max \left\{\left(\frac{C T_{0}}{\delta}\right)^{\frac{1}{\gamma_{0}+1}}, t_{0}\right\}$ such that

$$
\int_{\Omega \cap\{u \leq M\}}\left(\int_{\Omega \cap\{u \geq K\}} \frac{G(y, u)}{|x-y|^{\mu}} d y\right) G(x, u) d x \leq \delta .
$$

From $(g 3)$, we get

$$
\begin{aligned}
& \int_{\Omega \cap\left\{u_{k} \leq M\right\}}\left(\int_{\Omega \cap\left\{u_{k} \geq K\right\}} \frac{G\left(y, u_{k}\right)}{|x-y|^{\mu}} d y\right) G\left(x, u_{k}\right) d x \\
& \leq \frac{1}{K^{\gamma_{0}+1}} \int_{\Omega \cap\left\{u_{k} \leq M\right\}}\left(\int_{\Omega \cap\left\{u_{k} \geq K\right\}} \frac{u_{k}^{\gamma_{0}+1}(y) G\left(y, u_{k}\right)}{|x-y|^{\mu}} d y\right) G\left(x, u_{k}\right) d x \\
& \leq \frac{T_{0}}{K^{\gamma_{0}+1}} \int_{\Omega \cap\left\{u_{k} \leq M\right\}}\left(\int_{\Omega \cap\left\{u_{k} \geq K\right\}} \frac{u_{k}(y) g\left(y, u_{k}\right)}{|x-y|^{\mu}} d y\right) G\left(x, u_{k}\right) d x \\
& \leq \frac{T_{0}}{K^{\gamma_{0}+1}} \int_{\Omega}\left(\int_{\Omega} \frac{G\left(y, u_{k}\right)}{|x-y|^{\mu}} d y\right) g\left(x, u_{k}\right) u_{k} d x \leq \delta .
\end{aligned}
$$

Thus we have proved that

$$
\begin{aligned}
& \mid \int_{\Omega \cap\{u \leq M\}}\left(\int_{\Omega \cap\{u \geq K\}} \frac{G(y, u)}{|x-y|^{\mu}} d y\right) G(x, u) d x \\
& \quad-\int_{\Omega \cap\left\{u_{k} \leq M\right\}}\left(\int_{\Omega \cap\left\{u_{k} \geq K\right\}} \frac{G\left(y, u_{k}\right)}{|x-y|^{\mu}} d y\right) G\left(x, u_{k}\right) d x \mid \leq 2 \delta
\end{aligned}
$$

Finally, to complete the proof of Lemma, we need to verify that as $k \rightarrow \infty$

$$
\begin{gathered}
\mid \int_{\Omega \cap\left\{u_{k} \leq M\right\}}\left(\int_{\Omega \cap\left\{u_{k} \leq K\right\}} \frac{G\left(y, u_{k}\right)}{|x-y|^{\mu}} d y\right) G\left(x, u_{k}\right) d x- \\
\int_{\Omega \cap\{u \leq M\}}\left(\int_{\Omega \cap\{u \leq K\}} \frac{G(y, u)}{|x-y|^{\mu}} d y\right) G(x, u) d x \mid \rightarrow 0
\end{gathered}
$$

for fixed positive $K$ and $M$. It is easy to see that

$$
\left(\int_{\Omega \cap\left\{u_{k} \leq K\right\}} \frac{G\left(y, u_{k}\right)}{|x-y|^{\mu}} d y\right) G\left(x, u_{k}\right) \chi_{\Omega \cap\left\{u_{k} \leq M\right\}} \rightarrow\left(\int_{\Omega \cap\{u \leq K\}} \frac{G(y, u)}{|x-y|^{\mu}} d y\right) G(x, u) \chi_{\Omega \cap\{u \leq M\}}
$$

pointwise a.e. as $k \rightarrow \infty$. Now choose $r=\alpha$ in (3.1), which gives us that there exist a constant $C_{M, K}>0$ depending on $M$ and $K$ such that

$$
\begin{aligned}
& \int_{\Omega \cap\left\{u_{k} \leq M\right\}}\left(\int_{\Omega \cap\left\{u_{k} \leq K\right\}} \frac{G\left(y, u_{k}\right)}{|x-y|^{\mu}} d y\right) G\left(x, u_{k}\right) d x \\
& \leq C_{M, K} \int_{\Omega \cap\left\{u_{k} \leq M\right\}}\left(\int_{\left\{u_{k} \leq K\right\}} \frac{\left|u_{k}(y)\right|^{r}}{|x-y|^{\mu}} d y\right)\left|u_{k}(x)\right|^{r} d x \\
& \leq C_{M, K} \int_{\Omega} \int_{\Omega}\left(\frac{\left|u_{k}(y)\right|^{r}}{|x-y|^{\mu}} d y\right)\left|u_{k}(x)\right|^{r} d x \\
& \leq C_{M, K} C(n, \mu)\left\|u_{k}\right\|_{L^{\frac{2 n r}{2 n-\mu}}(\Omega)}^{2 r} \rightarrow C_{M, K} C(n, \mu)\|u\|_{L^{\frac{2 n r}{2 n-\mu}}(\Omega)}^{2 r} \text { as } k \rightarrow \infty,
\end{aligned}
$$


where we used the Hardy-Littlewood-Sobolev inequality in the last inequality and then used the fact that $u_{k} \rightarrow u$ strongly in $L^{q}(\Omega)$ for each $q \in[1, \infty)$. This implies that, using Theorem 4.9 of [4], there exists a constant $h \in L^{1}(\Omega)$ such that, up to a subsequence, for each $k$

$$
\left|\left(\int_{\Omega \cap\left\{u_{k} \leq K\right\}} \frac{G\left(y, u_{k}\right)}{|x-y|^{\mu}} d y\right) G\left(x, u_{k}\right) \chi_{\Omega \cap\left\{u_{k} \leq M\right\}}\right| \leq|h(x)|
$$

This helps us to employ the Lebesgue dominated convergence theorem and conclude (3.21).

Lemma 3.5 Let $\left\{u_{k}\right\} \subset X_{0}$ be a Palais Smale sequence of $J$. Then there exists a $u \in X_{0}$ such that, up to a subsequence, for all $\phi \in X_{0}$

$$
\int_{\Omega}\left(\int_{\Omega} \frac{G\left(y, u_{k}\right)}{|x-y|^{\mu}} d y\right) g\left(x, u_{k}\right) \phi d x \rightarrow \int_{\Omega}\left(\int_{\Omega} \frac{G(y, u)}{|x-y|^{\mu}} d y\right) g(x, u) \phi d x \text { as } k \rightarrow \infty .
$$

Proof. As we argued in previous Lemma, we have that there exists a $u \in X_{0}$ such that, up to a subsequence, $u_{k} \rightarrow u$ weakly in $X_{0}, u_{k} \rightarrow u$ pointwise a.e. in $\mathbb{R}^{n},\left\|u_{k}\right\| \rightarrow \tau$ as $k \rightarrow \infty$, for some $\tau \geq 0$ and $u_{k} \rightarrow u$ strongly in $L^{q}(\Omega), q \in[1, \infty)$ as $k \rightarrow \infty$.

Let $\Omega^{\prime} \subset \subset \Omega$ and $\varphi \in C_{c}^{\infty}(\Omega)$ such that $0 \leq \varphi \leq 1$ and $\varphi \equiv 1$ in $\Omega^{\prime}$. Then by taking $\varphi$ as a test function in (3.5), we obtain the following estimate

$$
\begin{aligned}
& \int_{\Omega^{\prime}}\left(\int_{\Omega} \frac{G\left(y, u_{k}\right)}{|x-y|^{\mu}} d y\right) g\left(x, u_{k}\right) d x \leq \int_{\Omega}\left(\int_{\Omega} \frac{G\left(y, u_{k}\right)}{|x-y|^{\mu}} d y\right) g\left(x, u_{k}\right) \varphi d x \\
& \leq \epsilon_{k}\|\varphi\|+M\left(\left\|u_{k}\right\|^{\frac{n}{s}}\right) \int_{\mathbb{R}^{2 n}} \frac{\left|u_{k}(x)-u_{k}(y)\right|^{\frac{n}{s}-2}\left(u_{k}(x)-u_{k}(y)\right)(\varphi(x)-\varphi(y))}{|x-y|^{2 n}} d x d y \\
& \leq \epsilon_{k}\|\varphi\|+C\left\|u_{k}\right\|\|\varphi\| \leq C,
\end{aligned}
$$

since $\left\|u_{k}\right\| \leq C_{0}$ for all $k$. This implies that the sequence $\left\{\mu_{k}\right\}:=\left\{\left(\int_{\Omega} \frac{G\left(y, u_{k}\right)}{x-\left.y\right|^{\mu}} d y\right) g\left(x, u_{k}\right)\right\}$ is bounded in $L_{\text {loc }}^{1}(\Omega)$ which implies that up to a subsequence, $\mu_{k} \rightarrow \mu$ in the weak ${ }^{*}$-topology as $k \rightarrow \infty$, where $\mu$ denotes a Radon measure. So for any $\phi \in C_{c}^{\infty}(\Omega)$ we get

$$
\lim _{k \rightarrow \infty} \int_{\Omega}\left(\int_{\Omega} \frac{G\left(y, u_{k}\right)}{|x-y|^{\mu}} d y\right) g\left(x, u_{k}\right) \phi d x=\int_{\Omega} \phi d \mu, \forall \phi \in C_{c}^{\infty}(\Omega) .
$$

Since $u_{k}$ satisfies (3.5), for any measurable set $E \subset \Omega$, taking $\phi \in C_{c}^{\infty}(\Omega)$ such that $\operatorname{supp} \phi \subset$ $E$, we get that

$$
\begin{aligned}
\mu(E) & =\int_{E} \phi d \mu=\lim _{k \rightarrow \infty} \int_{E} \int_{\Omega}\left(\frac{G\left(y, u_{k}\right)}{|x-y|^{\mu}} d y\right) g\left(x, u_{k}\right) \phi(x) d x \\
& =\lim _{k \rightarrow \infty} \int_{\Omega} \int_{\Omega}\left(\frac{G\left(y, u_{k}\right)}{|x-y|^{\mu}} d y\right) g\left(x, u_{k}\right) \phi(x) d x \\
& =\lim _{k \rightarrow \infty} M\left(\left\|u_{k}\right\|^{\frac{n}{s}}\right) \int_{\mathbb{R}^{n}} \int_{\mathbb{R}^{n}} \frac{\left|u_{k}(x)-u_{k}(y)\right|^{\frac{n}{s}-2}\left(u_{k}(x)-u_{k}(y)\right)(\phi(x)-\phi(y))}{|x-y|^{2 n}} d x d y \\
& =M\left(\tau^{\frac{n}{s}}\right) \int_{\mathbb{R}^{n}} \int_{\mathbb{R}^{n}} \frac{|u(x)-u(y)|^{\frac{n}{s}-2}(u(x)-u(y))(\phi(x)-\phi(y))}{|x-y|^{2 n}} d x d y,
\end{aligned}
$$


where we used the continuity of $M$ and weak convergence of $u_{k}$ to $u$ in $X_{0}$. This implies that $\mu$ is absolutely continuous with respect to the Lebesgue measure. Thus, Radon-Nikodym theorem establishes that there exists a function $h \in L_{\text {loc }}^{1}(\Omega)$ such that for any $\phi \in C_{c}^{\infty}(\Omega)$, $\int_{\Omega} \phi d \mu=\int_{\Omega} \phi h d x$. Therefore for any $\phi \in C_{c}^{\infty}(\Omega)$ we get

$$
\lim _{k \rightarrow \infty} \int_{\Omega}\left(\int_{\Omega} \frac{G\left(y, u_{k}\right)}{|x-y|^{\mu}} d y\right) g\left(x, u_{k}\right) \phi d x=\int_{\Omega} \phi h d x=\int_{\Omega}\left(\int_{\Omega} \frac{G(y, u)}{|x-y|^{\mu}} d y\right) g(x, u) \phi d x
$$

and the above holds for any $\phi \in X_{0}$ using the density argument. This completes the proof. Now we define the Nehari manifold associated to the functional $J$, as

$$
\mathcal{N}:=\left\{0 \not \equiv u \in X_{0}:\left\langle J^{\prime}(u), u\right\rangle=0\right\}
$$

and let $b:=\inf _{u \in \mathcal{N}} J(u)$. Then we need the following Lemma to compare $c_{*}$ and $b$.

Lemma 3.6 If condition ( $g 4)$ holds, then for each $x \in \Omega, \operatorname{tg}(x, t)-\frac{\gamma n}{2 s} G(x, t)$ is increasing for $t \geq 0$. In particular $t g(x, t)-\frac{\gamma n}{2 s} G(x, t) \geq 0$ for all $(x, t) \in \Omega \times[0, \infty)$ which implies $\frac{G(x, t)}{t^{\frac{\gamma}{2 s}}}$ is non-decreasing for $t>0$.

Proof. Suppose $0<t<r$. Then for each $x \in \Omega$, we obtain

$$
\begin{aligned}
\operatorname{tg}(x, t)-\frac{\gamma n}{2 s} G(x, t) & =\frac{g(x, t)}{t^{l}} t^{l+1}-\frac{\gamma n}{2 s} G(x, r)+\frac{\gamma n}{2 s} \int_{t}^{r} g(x, \tau) d \tau \\
& <\frac{g(x, t)}{t^{l}} t^{l+1}-\frac{\gamma n}{2 s} G(x, r)+\frac{\gamma n}{2 s(l+1)} \frac{g(x, r)}{r^{l}}\left(r^{l+1}-t^{l+1}\right) \\
& \leq r g(x, r)-\frac{\gamma n}{2 s} G(x, r),
\end{aligned}
$$

using ( $g 4)$. This completes the proof.

Lemma 3.7 Under the assumptions (M2) and (g4), it holds $c_{*} \leq b$.

Proof. Let $u \in \mathcal{N}$ be non negative and we define $h:(0, \infty) \rightarrow \mathbb{R}$ by $h(t)=J(t u)$. Then for all $t>0$

$$
h^{\prime}(t)=\left\langle J^{\prime}(t u), u\right\rangle=M\left(t^{\frac{n}{s}}\|u\|^{\frac{n}{s}}\right) t^{\frac{n}{s}-1}\|u\|^{\frac{n}{s}}-\int_{\Omega}\left(\int_{\Omega} \frac{G(y, t u)}{|x-y|^{\mu}} d y\right) g(x, t u) u d x .
$$

Since $\left\langle J^{\prime}(u), u\right\rangle=0$ and $t \mapsto \frac{g(x, t)}{t^{\frac{\gamma n}{2 s}-1}}$ is increasing for $t>0$, we have

$$
\begin{aligned}
& h^{\prime}(t)=\|u\|^{\frac{\gamma n}{s}} t^{\frac{\gamma n}{s}}-1\left(\frac{M\left(t^{\frac{n}{s}}\|u\|^{\frac{n}{s}}\right)}{t^{(\gamma-1) \frac{n}{s}}\|u\|^{(\gamma-1) \frac{n}{s}}}-\frac{M\left(\|u\|^{\frac{n}{s}}\right)}{\|u\|^{(\gamma-1) \frac{n}{s}}}\right) \\
& +t^{\frac{\gamma n}{s}-1} \int_{\Omega}\left(\int_{\Omega} \frac{\frac{G(y, u) g(x, u)}{u^{\frac{\gamma n}{2 s}-1}(x)}}{|x-y|^{\mu}} d y-\int_{\Omega} \frac{\frac{G(y, t u) g(x, t u)}{(t u)^{\frac{1 n}{2 s}-1}(x) \frac{t^{\frac{\eta n}{2}}}{2 s}}}{|x-y|^{\mu}} d y\right) u^{\frac{\gamma n}{2 s}}(x) d x \\
& \geq\|u\|^{\frac{\gamma n}{s}} t^{\frac{\gamma n}{s}-1}\left(\frac{M\left(t^{\frac{n}{s}}\|u\|^{\frac{n}{s}}\right)}{t^{\frac{(\gamma-1) n}{s}}\|u\|^{\frac{(\gamma-1) n}{s}}}-\frac{M\left(\|u\|^{\frac{n}{s}}\right)}{\|u\|^{\frac{(\gamma-1) n}{s}}}\right) \\
& +t^{\frac{\gamma n}{s}-1} \int_{\Omega}\left(\int_{\Omega}\left(G(y, u)-\frac{G(y, t u)}{t^{\frac{\gamma n}{2 s}}}\right) \frac{1}{|x-y|^{\mu}} d y\right) \frac{g(x, t u)}{(t u)^{\frac{\gamma n}{2 s}-1}(x)} u^{\frac{\gamma n}{2 s}}(x) d x .
\end{aligned}
$$


when $0<t<1$. So using Lemma 3.6 and $(M 2)$ we have $h^{\prime}(1)=0, h^{\prime}(t) \geq 0$ for $0<t<1$ and $h^{\prime}(t)<0$ for $t>1$. Hence $J(u)=\max _{t \geq 0} J(t u)$. Now define $f:[0,1] \rightarrow X_{0}$ as $f(t)=\left(t_{0} u\right) t$, where $t_{0}>1$ is such that $J\left(t_{0} u\right)<0$. Then we have $f \in \Gamma$ and therefore

$$
c_{*} \leq \max _{t \in[0,1]} J(f(t)) \leq \max _{t \geq 0} J(t u)=J(u) \leq \inf _{u \in \mathcal{N}} J(u)=b .
$$

Hence the proof is complete.

Definition 3.8 A solution $u_{0}$ of $(\mathcal{M})$ is a ground state if $u_{0}$ is a weak solution of $(\mathcal{M})$ and satisfies $J\left(u_{0}\right)=\inf _{u \in \mathcal{N}} J(u)$.

Since $c_{*} \leq b$ in order to obtain a ground state solution $u_{0}$ for $(\mathcal{M})$, it is enough to show that there exists a weak solution of $(\mathcal{M})$ such that $J\left(u_{0}\right)=c_{*}$.

Lemma 3.9 Any nontrivial solution of problem $(\mathcal{M})$ is nonnegative.

Proof. Let $u \in X_{0} \backslash\{0\}$ be a critical point of functional $J$. Clearly $u^{-}=\max \{-u, 0\} \in X_{0}$. Then $\left\langle J^{\prime}(u), u^{-}\right\rangle=0$, i.e.

$$
\begin{gathered}
M\left(\|u\|^{\frac{n}{s}}\right) \int_{\mathbb{R}^{2 n}} \frac{|u(x)-u(y)|^{\frac{n}{s}-2}(u(x)-u(y))\left(u^{-}(x)-u^{-}(y)\right)}{|x-y|^{2 n}} d x d y \\
=\int_{\Omega}\left(\int_{\Omega} \frac{G(y, u)}{|x-y|^{\mu}} d y\right) g(x, u) u^{-} d x .
\end{gathered}
$$

For a.e. $x, y \in \mathbb{R}^{n}$, using $\left|u^{-}(x)-u^{-}(y)\right| \leq|u(x)-u(y)|$, we have

$$
\begin{aligned}
& |u(x)-u(y)|^{\frac{n}{s}-2}(u(x)-u(y))\left(u^{-}(x)-u^{-}(y)\right) \\
& =-|u(x)-u(y)|^{\frac{n}{s}-2}\left(u^{+}(x) u^{-}(y)+u^{-}(x) u^{+}(y)+\left|u^{-}(x)-u^{-}(y)\right|^{2}\right) \\
& \leq-\left|u^{-}(x)-u^{-}(y)\right|^{\frac{n}{s}}
\end{aligned}
$$

and $g(x, u) u^{-}=0$ a.e. $x \in \Omega$ by assumption. Hence,

$$
0 \leq-M\left(\|u\|^{\frac{n}{s}}\right)\left\|u^{-}\right\|^{\frac{n}{s}} \leq 0
$$

So, $u^{-} \equiv 0$ since $\|u\|>0$ and (M3) holds. Hence $u \geq 0$ a.e. in $\Omega$.

Proof of Theorem 2.6; Since $J$ satisfies the Mountain Pass geometry (refer Lemma 3.1), by Mountain Pass Lemma we know that there exists a Palais Smale $\left\{u_{k}\right\}$ sequence for $J$ at $c_{*}$. Then by Lemma 3.2 $\left\{u_{k}\right\}$ must be bounded in $X_{0}$ so that, up to a subsequence, $u_{k} \rightarrow u_{0}$ weakly in $X_{0}$, strongly in $L^{q}(\Omega)$ for $q \in[1, \infty)$, pointwise a.e. in $\Omega$, for some $u_{0} \in X_{0}$ and $\left\|u_{k}\right\| \rightarrow \rho_{0} \geq 0$ as $k \rightarrow \infty$.

Claim 1: $u_{0} \not \equiv 0$ in $\Omega$.

Proof. We argue by contradiction. Suppose that $u_{0} \equiv 0$. Then using Lemma 3.4, we obtain

$$
\int_{\Omega}\left(\int_{\Omega} \frac{G\left(y, u_{k}\right)}{|x-y|^{\mu}} d y\right) G\left(x, u_{k}\right) d x \rightarrow 0 \text { as } k \rightarrow \infty .
$$


This together with $\lim _{k \rightarrow \infty} J\left(u_{k}\right)=c_{*}$ gives that

$$
\lim _{k \rightarrow \infty} \frac{s}{n} \hat{M}\left(\left\|u_{k}\right\|^{\frac{n}{s}}\right)=c_{*}<\frac{s}{n} \hat{M}\left(\left(\frac{2 n-\mu}{2 n} \alpha_{n, s}\right)^{\frac{n-s}{s}}\right) .
$$

Thus $\hat{M}$ being increasing function gives that there exists a $k_{0} \in \mathbb{N}$ such that $\left\|u_{k}\right\|^{\frac{n}{s}} \leq$ $\left(\frac{2 n-\mu}{2 n} \alpha_{n, s}\right)^{\frac{n-s}{s}}$ for all $k \geq k_{0}$. We fix $k \geq k_{0}$ and choose $p>1$ close to 1 and $\epsilon>0$ small enough such that

$$
\frac{2 n p(1+\epsilon)}{2 n-\mu}\left\|u_{k}\right\|^{\frac{n}{n-s}}<\alpha_{n, s} .
$$

Using the growth assumptions on $g$ and Theorem 1.2 we have

$$
\begin{aligned}
&\left\|g\left(\cdot, u_{k}\right) u_{k}\right\|_{L^{\frac{2 n}{2 n-\mu}(\Omega)}}^{\frac{2 n-\mu}{2 n}} \leq C(\epsilon)\left(\int_{\Omega}\left|u_{k}\right|^{\frac{2 n \alpha}{2 n-\mu}} d x+\int_{\Omega}\left|u_{k}\right|^{\frac{2 n r}{2 n-\mu}} \exp \left(\frac{2 n(1+\epsilon)}{2 n-\mu}\left|u_{k}\right|^{\frac{n}{n-s}}\right) d x\right) \\
& \leq C(\epsilon)\left(\int_{\Omega}\left|u_{k}\right|^{\frac{2 n \alpha}{2 n-\mu}} d x+\left(\int_{\Omega}\left|u_{k}\right|^{\frac{2 n r p^{\prime}}{2 n-\mu}} d x\right)^{\frac{1}{p^{\prime}}}\right. \\
&\left.\left(\int_{\Omega} \exp \left(\frac{2 n p(1+\epsilon)}{2 n-\mu}\left\|u_{k}\right\|^{\frac{n}{n-s}}\left(\frac{\left|u_{k}\right|}{\left\|u_{k}\right\|}\right)^{\frac{n}{n-s}}\right) d x\right)^{\frac{1}{p}}\right)
\end{aligned}
$$

where $1<\alpha<l+1$ and $1<r$. Thus,

$$
\left\|g\left(\cdot, u_{k}\right) u_{k}\right\|_{L^{\frac{2 n}{2 n-\mu}}(\Omega)} \leq C(\epsilon)\left(\left\|u_{k}\right\|_{L^{\frac{2 n \alpha}{2 n-\mu}(\Omega)}}^{\frac{2 n-\mu}{2 n \alpha}}+\left\|u_{k}\right\|_{L^{\frac{2 n r p^{\prime}}{2 n-\mu}}(\Omega)}^{\frac{2 n-\mu}{2 n r}}\right) \rightarrow 0 \text { as } k \rightarrow \infty,
$$

where $p^{\prime}$ denotes the Hölder conjugate of $p$ and $C(\epsilon)>0$ is a constant depending on $\epsilon$ which may change value at each step. From the semigroup property of the Riesz potential and Hardy-Littlewood-Sobolev inequality we get that

$$
\begin{aligned}
& \left|\int_{\Omega}\left(\int_{\Omega} \frac{G\left(y, u_{k}\right)}{|x-y|^{\mu}} d y\right) g\left(x, u_{k}\right) u_{k} d x\right| \\
& \leq\left(\int_{\Omega}\left(\int_{\Omega} \frac{G\left(y, u_{k}\right)}{|x-y|^{\mu}} d y\right) G\left(x, u_{k}\right) d x\right)^{\frac{1}{2}}\left(\int_{\Omega}\left(\int_{\Omega} \frac{g\left(y, u_{k}\right) u_{k}}{|x-y|^{\mu}} d y\right) g\left(x, u_{k}\right) u_{k} d x\right)^{\frac{1}{2}} \\
& \leq\left(\int_{\Omega}\left(\int_{\Omega} \frac{G\left(y, u_{k}\right)}{|x-y|^{\mu}} d y\right) G\left(x, u_{k}\right) d x\right)^{\frac{1}{2}} C_{n, \mu}\left\|g\left(\cdot, u_{k}\right) u_{k}\right\|_{L^{\frac{2 n}{2 n-\mu}}(\Omega)} \rightarrow 0
\end{aligned}
$$

as $k \rightarrow \infty$ using (3.23) and (3.24). This together with $\left\langle J^{\prime}\left(u_{k}\right), u_{k}\right\rangle=0$ implies that $M\left(\left\|u_{k}\right\|^{\frac{n}{s}}\right)\left\|u_{k}\right\|^{\frac{n}{s}} \rightarrow 0$. From $(M 3)$, we deduce that $\left\|u_{k}\right\| \rightarrow 0$. Furthermore, we obtain $\lim _{k \rightarrow \infty} J\left(u_{k}\right)=0=c_{*}$, which is a contradiction to the fact that $c_{*}>0$. Hence, we must have $u_{0} \not \equiv 0$.

Claim 2: $M\left(\left\|u_{0}\right\|^{\frac{n}{s}}\right)\left\|u_{0}\right\|^{\frac{n}{s}} \geq \int_{\Omega}\left(\int_{\Omega} \frac{G\left(y, u_{0}\right)}{|x-y|^{\mu}} d y\right) g\left(x, u_{0}\right) u_{0} d x$.

Proof. Suppose by contradiction that $M\left(\left\|u_{0}\right\|^{\frac{n}{s}}\right)\left\|u_{0}\right\|^{\frac{n}{s}}<\int_{\Omega}\left(\int_{\Omega} \frac{G\left(y, u_{0}\right)}{|x-y|^{\mu}} d y\right) g\left(x, u_{0}\right) u_{0} d x$.

That is, $\left\langle J^{\prime}\left(u_{0}\right), u_{0}\right\rangle<0$. 
It is easy to see, using $(M 2)$, that $M(t) t \geq M(1) t^{\gamma}$ when $t \in[0,1]$. So for $0<t<\frac{1}{\left\|u_{0}\right\|}$, using Lemma 3.6 and Hardy-Littlewood-Sobolev inequality we have that

$$
\begin{aligned}
\left\langle J^{\prime}\left(t u_{0}\right), u_{0}\right\rangle & \geq M\left(t^{\frac{n}{s}}\left\|u_{0}\right\|^{\frac{n}{s}}\right) t^{\frac{n}{s}-1}\left\|u_{0}\right\|^{\frac{n}{s}}-\frac{2 s}{\gamma n} \int_{\Omega}\left(\int_{\Omega} \frac{g\left(y, t u_{0}\right) t u_{0}(y)}{|x-y|^{\mu}} d y\right) g\left(x, t u_{0}\right) u_{0}(x) d x \\
& \geq M(1) t^{\frac{\gamma n}{s}-1}\left\|u_{0}\right\|^{\frac{\gamma n}{s}}-\frac{C}{t}\left(\int_{\Omega}\left|g\left(x, t u_{0}\right) t u_{0}\right|^{\frac{2 n}{2 n-\mu}} d x\right)^{\frac{2 n-\mu}{n}} .
\end{aligned}
$$

But from the growth assumptions on $g$ we already know that for $\epsilon>0, \alpha>\frac{\gamma n}{2 s}$ and $r>\frac{\gamma n}{2 s}$,

$$
\begin{aligned}
& \left(\int_{\Omega}\left|g\left(x, t u_{0}\right) t u_{0}\right|^{\frac{2 n}{2 n-\mu}} d x\right)^{\frac{2 n-\mu}{n}} \\
& \leq C(\epsilon)\left(\int_{\Omega}\left|t u_{0}\right|^{\frac{2 n \alpha}{2 n-\mu}}+\left\|t u_{0}\right\|^{\frac{2 r n}{2 n-\mu}}\left(\int_{\Omega} \exp \left(\frac{4 n(1+\epsilon)\left\|t u_{0}\right\|^{\frac{n}{n-s}}}{2 n-\mu}\left(\frac{\left|t u_{0}\right|}{\left\|t u_{0}\right\|}\right)^{\frac{n}{n-s}}\right)\right)^{\frac{1}{2}}\right)^{\frac{2 n-\mu}{n}} \\
& \leq C(\epsilon)\left(\left\|t u_{0}\right\|^{2 \alpha}+\left\|t u_{0}\right\|^{2 r}\right)
\end{aligned}
$$

by choosing $t<\left(\frac{(2 n-\mu) \alpha_{n, s}}{4 n(1+\epsilon)\left\|u_{0}\right\|^{\frac{n}{n-s}}}\right)^{\frac{n-s}{n}}$ and using Trudinger-Moser inequality. Therefore for $t>0$ small enough as above, we obtain

$$
\left\langle J^{\prime}\left(t u_{0}\right), u_{0}\right\rangle \geq M(1) t^{\frac{\gamma n}{s}-1}\left\|u_{0}\right\|^{\frac{\gamma n}{s}}-C(\epsilon)\left(t^{2 \alpha-1}\left\|u_{0}\right\|^{2 \alpha}+t^{2 r-1}\left\|u_{0}\right\|^{2 r}\right)
$$

which suggests that $\left\langle J^{\prime}\left(t u_{0}\right), u_{0}\right\rangle>0$ when $t$ is sufficiently small. Thus there exists a $\sigma \in(0,1)$ such that $\left\langle J^{\prime}\left(\sigma u_{0}\right), u_{0}\right\rangle=0$ that is, $\sigma u_{0} \in \mathcal{N}$. Thus from Lemmas 3.6, 3.7 and Remark 2.1, it follows that

$$
\begin{aligned}
& c_{*} \leq b \leq J\left(\sigma u_{0}\right)=J\left(\sigma u_{0}\right)-\frac{s}{n \gamma}\left\langle J^{\prime}\left(\sigma u_{0}\right), \sigma u_{0}\right\rangle \\
& =\frac{s}{n} \hat{M}\left(\left\|\sigma u_{0}\right\|^{\frac{n}{s}}\right)-\frac{s M\left(\left\|\sigma u_{0}\right\|^{\frac{n}{s}}\right)\left\|\sigma u_{0}\right\|^{\frac{n}{s}}}{n \gamma}+\frac{s}{n \gamma} \int_{\Omega}\left(\int_{\Omega} \frac{G\left(y, \sigma u_{0}\right)}{|x-y|^{\mu}} d y\right)\left(g\left(x, \sigma u_{0}\right) \sigma u_{0}-\frac{n \gamma}{2 s} G\left(x, \sigma u_{0}\right)\right) \\
& <\frac{s}{n} \hat{M}\left(\left\|u_{0}\right\|^{\frac{n}{s}}\right)-\frac{s}{n \gamma} M\left(\left\|u_{0}\right\|^{\frac{n}{s}}\right)\left\|u_{0}\right\|^{\frac{n}{s}}+\frac{s}{n \gamma} \int_{\Omega}\left(\int_{\Omega} \frac{G\left(y, u_{0}\right)}{|x-y|^{\mu}} d y\right)\left(g\left(x, u_{0}\right) u_{0}-\frac{n \gamma}{2 s} G\left(x, u_{0}\right)\right) d x .
\end{aligned}
$$

Also by lower semicontinuity of norm and Fatou's Lemma, we obtain

$$
\begin{aligned}
c_{*} \leq b< & \liminf _{k \rightarrow \infty}\left(\frac{s}{n} \hat{M}\left(\left\|u_{k}\right\|^{\frac{n}{s}}\right)-\frac{s}{n \gamma} M\left(\left\|u_{k}\right\|^{\frac{n}{s}}\right)\left\|u_{k}\right\|^{n^{\frac{n}{s}}}\right) \\
& +\liminf _{k \rightarrow \infty} \frac{s}{n \gamma} \int_{\Omega}\left(\int_{\Omega} \frac{G\left(y, u_{k}\right)}{|x-y|^{\mu}} d y\right)\left[g\left(x, u_{k}\right) u_{k}-\frac{n \gamma}{2 s} G\left(x, u_{k}\right)\right] d x \\
\leq & \lim _{k \rightarrow \infty}\left[J\left(u_{k}\right)-\frac{s}{n \gamma}\left\langle J^{\prime}\left(u_{k}\right), u_{k}\right\rangle\right]=c_{*},
\end{aligned}
$$

which is a contradiction. Hence Claim 2 is proved.

Claim 3: $J\left(u_{0}\right)=c_{*}$.

Proof. Using $\int_{\Omega}\left(\int_{\Omega} \frac{G\left(y, u_{k}\right)}{|x-y|^{\mu}} d y\right) G\left(x, u_{k}\right) d x \rightarrow \int_{\Omega}\left(\int_{\Omega} \frac{G\left(y, u_{0}\right)}{|x-y|^{\mu}} d y\right) G\left(x, u_{0}\right) d x$ and lower 
semicontinuity of norm we have $J\left(u_{0}\right) \leq c_{*}$. Now we are going to show that the case $J\left(u_{0}\right)<c_{*}$ can not occur. Indeed, if $J\left(u_{0}\right)<c_{*}$ then $\left\|u_{0}\right\|^{\frac{n}{s}}<\rho_{0}^{\frac{n}{s}}$. Moreover,

$$
\frac{s}{n} \hat{M}\left(\rho_{0}^{\frac{n}{s}}\right)=\lim _{k \rightarrow \infty} \frac{s}{n} \hat{M}\left(\left\|u_{k}\right\|^{\frac{n}{s}}\right)=c_{*}+\frac{1}{2} \int_{\Omega}\left(\int_{\Omega} \frac{G\left(y, u_{0}\right)}{|x-y|^{\mu}} d y\right) G\left(x, u_{0}\right) d x
$$

This gives that

$$
\rho_{0}^{\frac{n}{s}}=\hat{M}^{-1}\left(\frac{n}{s} c_{*}+\frac{n}{2 s} \int_{\Omega}\left(\int_{\Omega} \frac{G\left(y, u_{0}\right)}{|x-y|^{\mu}} d y\right) G\left(x, u_{0}\right) d x\right) .
$$

Next defining $v_{k}=\frac{u_{k}}{\left\|u_{k}\right\|}$ and $v_{0}=\frac{u_{0}}{\rho_{0}}$, we have $v_{k} \rightarrow v_{0}$ in $X_{0}$ and $\left\|v_{0}\right\|<1$. Thus by Lemma 2.5 ,

$$
\sup _{k \in \mathbb{N}} \int_{\Omega} \exp \left(p\left|v_{k}\right|^{\frac{n}{n-s}}\right) d x<\infty \text { for all } 1<p<\frac{\alpha_{n, s}}{\left(1-\left\|v_{0}\right\|^{\frac{n}{s}}\right)^{\frac{s}{n-s}}} .
$$

On the other hand, by Claim 2, (2.1) and Lemma 3.6, we have

$$
\begin{aligned}
J\left(u_{0}\right) \geq \frac{s}{n} \hat{M}\left(\left\|u_{0}\right\|^{\frac{n}{s}}\right)-\frac{s}{n \gamma} M\left(\left\|u_{0}\right\|^{\frac{n}{s}}\right)\left\|u_{0}\right\|^{\frac{n}{s}} \\
\quad+\frac{s}{n \gamma} \int_{\Omega}\left(\int_{\Omega} \frac{G\left(y, u_{0}\right)}{|x-y|^{\mu}} d y\right)\left(g\left(x, u_{0}\right) u_{0}-\frac{n \gamma}{2 s} G\left(x, u_{0}\right)\right) d x \geq 0 .
\end{aligned}
$$

Using this together with Lemma 3.3 and the equality, $\frac{n}{s}\left(c_{*}-J\left(u_{0}\right)\right)=\hat{M}\left(\rho_{0}^{\frac{n}{s}}\right)-\hat{M}\left(\left\|u_{0}\right\|^{\frac{n}{s}}\right)$ we obtain

$$
\hat{M}\left(\rho_{0}^{\frac{n}{s}}\right) \leq \frac{n}{s} c_{*}+\hat{M}\left(\left\|u_{0}\right\|^{\frac{n}{s}}\right)<\hat{M}\left(\left(\frac{2 n-\mu}{2 n} \alpha_{n, s}\right)^{\frac{n-s}{s}}\right)+\hat{M}\left(\left\|u_{0}\right\|^{\frac{n}{s}}\right)
$$

and therefore by $(M 1)$

$$
\rho_{0}^{\frac{n}{s}}<\hat{M}^{-1}\left(\hat{M}\left(\left(\frac{2 n-\mu}{2 n} \alpha_{n, s}\right)^{\frac{n-s}{s}}\right)+\hat{M}\left(\left\|u_{0}\right\|^{\frac{n}{s}}\right)\right) \leq\left(\frac{2 n-\mu}{2 n} \alpha_{n, s}\right)^{\frac{n-s}{s}}+\left\|u_{0}\right\|^{\frac{n}{s}} .
$$

Since $\rho_{0}^{\frac{n}{s}}\left(1-\left\|v_{0}\right\|^{\frac{n}{s}}\right)=\rho_{0}^{\frac{n}{s}}-\left\|u_{0}\right\|^{\frac{n}{s}}$, from (3.27) it follows that

$$
\rho_{0}^{\frac{n}{s}}<\frac{\left(\frac{2 n-\mu}{2 n} \alpha_{n, s}\right)^{\frac{n-s}{s}}}{1-\left\|v_{0}\right\|^{\frac{n}{s}}} .
$$

Thus, there exists $\beta>0$ such that $\left\|u_{k}\right\|^{\frac{n}{n-s}}<\beta<\frac{\alpha_{n, s}(2 n-\mu)}{2 n\left(1-\left\|v_{0}\right\|^{\frac{n}{s}}\right)^{\frac{s}{n-s}}}$ for $k$ large. We can choose $q>1$ close to 1 such that $q\left\|u_{k}\right\|^{\frac{n}{n-s}} \leq \beta<\frac{(2 n-\mu) \alpha_{n, s}}{2 n\left(1-\left\|v_{0}\right\|^{\frac{n}{s}}\right)^{\frac{s}{n-s}}}$ and using (3.26), we conclude that for $k$ large

$$
\int_{\Omega} \exp \left(\frac{2 n q\left|u_{k}\right|^{n / n-s}}{2 n-\mu}\right) d x \leq \int_{\Omega} \exp \left(\frac{2 n \beta\left|v_{k}\right|^{n / n-s}}{2 n-\mu}\right) d x \leq C .
$$


Let us recall (2.3) and (3.24) to get that

$$
\begin{aligned}
& \left|\int_{\Omega}\left(\int_{\Omega} \frac{G\left(y, u_{k}\right)}{|x-y|^{\mu}} d y\right) g\left(x, u_{k}\right) u_{k} d x\right| \leq C\left(\left\|u_{k}\right\|_{L^{\frac{2 n-\mu}{2 n-\mu}(\Omega)}}^{\frac{2 n-\mu}{2 n \alpha}}+\left\|u_{k}\right\|_{L^{\frac{2 n n q^{\prime}}{2 n-\mu}(\Omega)}}^{\frac{2 n-\mu}{2 n r}}\right) \\
& \rightarrow C\left(\left\|u_{0}\right\|_{L^{\frac{2 n \alpha}{2 n-\mu}}(\Omega)}^{\frac{2 n-\mu}{2 n \alpha}}+\left\|u_{0}\right\|_{L^{\frac{2 n r q^{\prime}}{2 n-\mu}}(\Omega)}^{\frac{2 n-\mu}{2 n r}}\right)
\end{aligned}
$$

as $k \rightarrow \infty$. Then the pointwise convergence of $\left(\int_{\Omega} \frac{G\left(y, u_{k}\right)}{|x-y|^{\mu}} d y\right) g\left(x, u_{k}\right) u_{k}$ to $\left(\int_{\Omega} \frac{G\left(y, u_{0}\right)}{|x-y|^{\mu}} d y\right) g\left(x, u_{0}\right) u_{0}$ as $k \rightarrow \infty$ asserts that

$$
\lim _{k \rightarrow \infty} \int_{\Omega}\left(\int_{\Omega} \frac{G\left(y, u_{k}\right)}{|x-y|^{\mu}} d y\right) g\left(x, u_{k}\right) u_{k} d x=\int_{\Omega}\left(\int_{\Omega} \frac{G\left(y, u_{0}\right)}{|x-y|^{\mu}} d y\right) g\left(x, u_{0}\right) u_{0} d x
$$

while using the Lebesgue dominated convergence theorem. Now Lemma 3.5, we get

$$
\int_{\Omega}\left(\int_{\Omega} \frac{G\left(y, u_{k}\right)}{|x-y|^{\mu}} d y\right) g\left(x, u_{k}\right)\left(u_{k}-u_{0}\right) d x \rightarrow 0 \text { as } k \rightarrow \infty .
$$

Since $\left\langle J^{\prime}\left(u_{k}\right), u_{k}-u_{0}\right\rangle \rightarrow 0$, it follows that

$$
M\left(\left\|u_{k}\right\|^{\frac{n}{s}}\right) \int_{\mathbb{R}^{2 n}} \frac{\left|u_{k}(x)-u_{k}(y)\right|^{\frac{n}{s}-2}\left(u_{k}(x)-u_{k}(y)\right)\left(\left(u_{k}-u_{0}\right)(x)-\left(u_{k}-u_{0}\right)(y)\right)}{|x-y|^{2 n}} d x d y \rightarrow 0 .
$$

We define $U_{k}(x, y)=u_{k}(x)-u_{k}(y)$ and $U_{0}(x, y)=u_{0}(x)-u_{0}(y)$ then using $u_{k} \rightarrow u_{0}$ weakly in $X_{0}$ and boundedness of $M\left(\left\|u_{k}\right\|^{\frac{n}{s}}\right)$, we have

$$
M\left(\left\|u_{k}\right\|^{\frac{n}{s}}\right) \int_{\mathbb{R}^{2 n}} \frac{\left|U_{0}(x, y)\right|^{\frac{n}{s}-2} U_{0}(x, y)\left(U_{k}(x, y)-U_{0}(x, y)\right)}{|x-y|^{2 n}} d x d y \rightarrow 0 \text { as } k \rightarrow \infty .
$$

Subtracting (3.29) from (3.28), we get

$M\left(\left\|u_{k}\right\|^{\frac{n}{s}}\right) \int_{\mathbb{R}^{2 n}} \frac{\left(\left|U_{k}(x, y)\right|^{\frac{n}{s}-2} U_{k}(x, y)-\left|U_{0}(x, y)\right|^{\frac{n}{s}-2} U_{0}(x, y)\right)\left(U_{k}(x, y)-U_{0}(x, y)\right)}{|x-y|^{2 n}} d x d y \rightarrow 0$

as $k \rightarrow \infty$. Now using this and the following inequality

$$
|a-b|^{p} \leq 2^{p-2}\left(|a|^{p-2} a-|b|^{p-2} b\right)(a-b) \text { for all } a, b \in \mathbb{R} \text { and } p \geq 2,
$$

with $a=u_{k}(x)-u_{k}(y)$ and $b=u_{0}(x)-u_{0}(y)$, we obtain

$$
M\left(\rho_{0}^{\frac{n}{s}}\right) \int_{\mathbb{R}^{2 n}} \frac{\left|U_{k}(x)-U_{0}(x)\right|^{\frac{n}{s}}}{|x-y|^{2 n}} d x d y \rightarrow 0 \text { as } k \rightarrow \infty .
$$

This implies that $u_{k} \rightarrow u$ strongly in $X_{0}$ and hence $J(u)=c_{*}$ which is a contradiction. Therefore, claim 3 holds true. Hence $J(u)=c_{*}=\lim _{k \rightarrow \infty} J\left(u_{k}\right)$ and $\left\|u_{k}\right\| \rightarrow \rho_{0}$ gives that $\rho_{0}=\left\|u_{0}\right\|$. Finally we have

$$
\begin{gathered}
M\left(\left\|u_{0}\right\|^{\frac{n}{s}}\right) \int_{\Omega} \frac{\left|u_{0}(x)-u_{0}(y)\right|^{\frac{n}{s}-2}\left(u_{0}(x)-u_{0}(y)\right)(\phi(x)-\phi(y))}{|x-y|^{2 n}} d x d y \\
=\int_{\Omega}\left(\int_{\Omega} \frac{G\left(y, u_{k}\right)}{|x-y|^{\mu}} d y\right) g\left(x, u_{0}\right) \phi d x
\end{gathered}
$$

for all $\phi \in X_{0}$. Thus, $u_{0}$ is a non trivial solution of $(\mathcal{M})$. By Lemma 3.9 we obtain that $u_{0}$ is the required nonnegative solution of $(\mathcal{M})$ which completes the proof. 
Acknowledgements: This research is supported by Science and Engineering Research Board, Department of Science and Technology, Government of India, Grant number: ECR/2017/002651. The second author wants to thank Bennett University for its hospitality during her visit there.

\section{References}

[1] Claudianor O. Alves, Daniele Cassani, Cristina Tarsi, and Minbo Yang. Existence and concentration of ground state solutions for a critical nonlocal Schrödinger equation in $\mathbb{R}^{2}$. J. Differential Equations, 261(3):1933-1972, 2016.

[2] Claudianor O. Alves and Minbo Yang. Existence of solutions for a nonlocal variational problem in $\mathbb{R}^{2}$ with exponential critical growth. J. Convex Anal., 24(4):1197-1215, 2017.

[3] R. Arora, J. Giacomoni, T. Mukherjee, and K. Sreenadh. n-Kirchhoff-Choquard equations with exponential nonlinearity. Nonlinear Anal., 108:113-144, 2019.

[4] Haim Brezis. Functional analysis, Sobolev spaces and partial differential equations. Universitext. Springer, New York, 2011.

[5] Eleonora Di Nezza, Giampiero Palatucci, and Enrico Valdinoci. Hitchhiker's guide to the fractional Sobolev spaces. Bull. Sci. Math., 136(5):521-573, 2012.

[6] J. Giacomoni, Pawan Kumar Mishra, and K. Sreenadh. Fractional elliptic equations with critical exponential nonlinearity. Adv. Nonlinear Anal., 5(1):57-74, 2016.

[7] J. Giacomoni, Pawan Kumar Mishra, and K. Sreenadh. Fractional Kirchhoff equation with critical exponential nonlinearity. Complex Var. Elliptic Equ., 61(9):1241-1266, 2016.

[8] Sarika Goyal and K. Sreenadh. Nehari manifold for non-local elliptic operator with concave-convex nonlinearities and sign-changing weight functions. Proc. Indian Acad. Sci. Math. Sci., 125(4):545-558, 2015.

[9] E. Parini L. Brasco, E. Lindgren. The fractional Cheeger problems. Interfaces Frr Bound., 16:419-458, 2014.

[10] M. Squassina L. Brasco, E. Parini. Stability of variatinal eigenvalues for the fractional p-laplacian. Discrete Contin. Dyn. Syst., 36:439-455, 2016.

[11] Fuyi Li, Chunjuan Gao, and Xiaoli Zhu. Existence and concentration of sign-changing solutions to Kirchhoff-type system with Hartree-type nonlinearity. J. Math. Anal. Appl., 448(1):60-80, 2017.

[12] E. H. Lieb. Existence and uniqueness of the minimizing solution of Choquard nonlinear equation. Stud. APPL. Math., 57:93-105, 1976/77. 
[13] Elliott H. Lieb and Michael Loss. Analysis, volume 14 of Graduate Studies in Mathematics. American Mathematical Society, Providence, RI, second edition, 2001.

[14] Dengfeng Lü. A note on Kirchhoff-type equations with Hartree-type nonlinearities. Nonlinear Anal., 99:35-48, 2014.

[15] Luca Martinazzi. Fractional Adams-Moser-Trudinger type inequalities. Nonlinear Anal., 127:263-278, 2015.

[16] Xiang Mingqi, Vicenţiu D. Rădulescu, and Binlin Zhang. Fractional Kirchhoff problems with critical Trudinger-Moser nonlinearity. Calc. Var. Partial Differential Equations, 58(2):Art. 57, 27, 2019.

[17] Vitaly Moroz and Jean Van Schaftingen. A guide to the Choquard equation. J. Fixed Point Theory Appl., 19(1):773-813, 2017.

[18] Enea Parini and Bernhard Ruf. On the Moser-Trudinger inequality in fractional SobolevSlobodeckij spaces. Atti Accad. Naz. Lincei Rend. Lincei Mat. Appl., 29(2):315-319, 2018.

[19] S. Pekar. Untersuchung über die Elektronentheorie der Kristalle. Akademie Verlag, Berlin. 1954.

[20] Kanishka Perera, Marco Squassina, and Yang Yang. Bifurcation and multiplicity results for critical fractional $p$-Laplacian problems. Mathematische Nachrichten, 289(2-3):332$342,2016$.

[21] P. Pucci, M. Xiang, and B. Zhang. Existence results for Schödinger-Choquard-Kirchhoff equations involving the fractional $p$-Laplacian. Adv. Calc. Var., 12(3):253-275, 2019.

[22] Raffaella Servadei and Enrico Valdinoci. Mountain pass solutions for non-local elliptic operators. J. Math. Anal. Appl., 389(2):887-898, 2012.

[23] M. Xiang, B. Zhang, and D Repovs. Existence and multiplicity of solutions for fractional Schrödinger-Kirchhoff equations with Trudinger-Moser nonlinearity. Nonlinear Anal., 186:74-98, 2018. 